Draft Version September 2, 2018

Preprint typeset using $\mathrm{LAT}_{\mathrm{E}} \mathrm{X}$ style emulateapj v. 12/16/11

\title{
HIDING IN PLAIN SIGHT: AN ABUNDANCE OF COMPACT MASSIVE SPHEROIDS IN THE LOCAL UNIVERSE
}

\author{
Alister W. Graham ${ }^{1}$, Bililign T. Dullo, Giulia A.D. Savorgnan \\ Centre for Astrophysics and Supercomputing, Swinburne University of Technology, Hawthorn, VIC, 3122, Australia. \\ Draft version September 2, 2018
}

\begin{abstract}
It has been widely remarked that compact, massive, elliptical-like galaxies are abundant at high redshifts but exceedingly rare in the Universe today, implying significant evolution such that their sizes at $z \sim 2 \pm 0.6$ have increased by factors of 3 to 6 to become today's massive elliptical galaxies. These claims have been based on studies which measured the half-light radii of galaxies as though they are all single component systems. Here we identify 21 spheroidal stellar systems within $90 \mathrm{Mpc}$ that have half-light, major-axis radii $R_{\mathrm{e}} \lesssim 2 \mathrm{kpc}$, stellar masses $0.7 \times 10^{11}<M_{*} / M_{\odot}<1.4 \times 10^{11}$, and Sérsic indices typically around a value of $n=2$ to 3 . This abundance of compact, massive spheroids in our own backyard - with a number density of $6.9 \times 10^{-6} \mathrm{Mpc}^{-3}$ (or $3.5 \times 10^{-5} \mathrm{Mpc}^{-3}$ per unit dex in stellar mass) - and with the same physical properties as the high-redshift galaxies, had been over-looked because they are encased in stellar disks which usually result in galaxy sizes notably larger than $2 \mathrm{kpc}$. Moreover, this number density is a lower limit because it has not come from a volume-limited sample. The actual density may be closer to $10^{-4}$, although further work is required to confirm this. We therefore conclude that not all massive 'spheroids' have undergone dramatic structural and size evolution since $z \sim 2 \pm 0.6$. Given that the bulges of local early-type disk galaxies are known to consist of predominantly old stars which existed at $z \sim 2$, it seems likely that some of the observed high redshift spheroids did not increase in size by building (3D) triaxial envelopes as commonly advocated, and that the growth of (2D) disks has also been important over the past $9-11$ billion years.
\end{abstract}

Subject headings: galaxies: bulges - galaxies: fundamental parameters - galaxies: evolution - galaxies: formation - galaxies: high-redshift

\section{INTRODUCTION}

A little over a decade ago it was advocated that the mass-size relation for massive galaxies $\left(M_{*}>0.2 \times\right.$ $\left.10^{11} h_{70}^{2} M_{\odot}\right)$ had evolved little from $z \sim 2.5$ to today (Trujillo et al. 2004). However this view was quickly challenged by Daddi et al. (2005), who had detected seven passively evolving galaxies at $z>1.4$, with stellar masses $\gtrsim 10^{11} h_{71}^{2} M_{\odot}$ and morphologies typical of elliptical/early-type galaxies. They questioned the lack of evolution because some of their galaxies had much smaller effective half light radii than local elliptical galaxies of the same mass (see also Papovich et al. 2005). They suggested this difference was either a real sign of galaxy size evolution, or artificial because of biasing active galactic nuclei light or morphological $K$-corrections due to blue cores in the high- $z$ sample. Addressing the latter uncertainty, Kriek et al. $(2006,2008)$ found that nearly half of their distant massive galaxies had old stellar populations and van Dokkum et al. (2008) subsequently showed that half of those had sizes less than $\sim 2$ kpc. Following Daddi et al. (2005), Trujillo et al. (2006a) reported galaxy sizes ranging from 1 to $5 \mathrm{kpc}$ for ten massive $\left(\sim 5 \times 10^{11} h_{70}^{2} M_{\odot}\right)$ galaxies at $1.2<z<1.7$. From this Trujillo et al. (2006a) concluded that (i) the sizes were at least a factor of 3 to 6 times lower than "the local counterparts" of similar mass, (ii) the structural properties of these high- $z$ objects are therefore rapidly changing, (iii) the data disagree with a scenario where

\footnotetext{
${ }^{1}$ Corresponding Author: AGraham@swin.edu.au
}

the more massive and passive galaxies are fully assembled by $1.2<z<1.7$ (i.e. a monolithic scenario), and (iv) they suggested that a dry merger scenario (no new star formation) was responsible for the subsequent evolution of these galaxies (see also Trujillo et al. 2007; Toft et al. 2007; Zirm et al. 2007; Buitrago et al. 2008; van Dokkum et al. 2008; Damjanov et al. 2009).

While not denying the occurrence of dry mergers, nor claiming that early-type galaxies had to have formed in a monolithic collapse (see Dekel \& Burkert 2014 and Zolotov et al. 2015 for an alternative), given the data under investigation we are questioning the universality of the above conclusions because they rest on the assumption as to what the "local counterparts" to the compact, massive high- $z$ galaxies actually are. Popular opinion has been that these distant objects are very rare today (e.g. Cimatti et al. 2008, and references therein), with Trujillo et al. (2009) reporting a local number density of $10^{-7} \mathrm{Mpc}^{-3}$, and Taylor et al. (2010) finding none and therefore concluding that the distant spheroidal stellar systems have experienced considerable size evolution to form today's massive elliptical galaxies. However, there are many independent lines of reasoning to think that this paradigm of early-type galaxy evolution may not be correct.

Key to much of this is that Trujillo et al. (2006a,b, 2007) used the sizes of $z \sim 0.1$ Sloan Digital Sky Survey (SDSS, York et al. 2000) early-type, i.e. elliptical and lenticular, galaxies (with Sérsic index $n>2.5$ ), to claim that there has been significant size, and surfacemass density, evolution of the distant spheroids (see also 
Taylor et al. 2010; Newman et al. 2012; McLure et al. 2013; Damjanov et al. 2014; Fang et al. 2015). In addition to early-type galaxies, there is however another type of spheroidal stellar system in the local universe, namely the bulges of disk galaxies, including the lenticular galaxies. Furthermore, there has been a slowly growing realization over the past three decades that most ${ }^{2}$ early-type galaxies infact consist of a bulge and a rotating stellar disk (e.g. Carter 1987; Capaccioli 1987, 1990; Nieto et al. 1988; D'Onofrio et al. 1995; Graham et al. 1998; Emsellem et al. 2011; Scott et al. 2014), arguing against the treatment of lenticular galaxies as single component systems. While many of these galaxies do not have massive bulges, and some contain pseudobulges built out of disk material (Kormendy \& Kennicutt 2004; see also Graham 2014 for cautionary remarks regarding their identification), our interest lies with some of the more massive lenticular and spiral galaxies that do have massive bulges. The most massive local bulges $\left(M_{*}>2 \times 10^{11} M_{\odot}\right)$ have sizes in excess of $2.5 \mathrm{kpc}$ are therefore not considered "compact"; as such they have been excluded from this study.

Here we effectively explore what evolutionary conclusion might have been drawn a decade ago for the compact massive spheroids at $z \approx 2$ if the size-mass comparison had of been performed with the massive bulges of present-day early-type disk galaxies rather than with the combined bulge+disk system. In so doing we can answer the question, Have the high- $z$ spheroidal stellar systems truly evolved to near extinction, or are they perhaps hiding in plain sight around us today? While we do not deny that there has been galaxy size evolution, we are suggesting a fundamentally different formation model which presents a dramatic shift in our way of thinking about how the compact massive objects at $z=2.0 \pm 0.6$ have actually evolved in our Universe. It is such that they have not expanded 3-6 times in size, nor accreted, through dry minor mergers, a three-dimesnional (3D) envelope of a similarly large dimension, to become today's massive elliptical galaxies (e.g. Hopkins et al. 2009; Carrasco et al. 2010; Cimatti et al. 2012; Fan et al. 2013b, De et al. 2014). Instead, we advocate the view presented by Graham (2013) that they have grown two-dimensional (2D) planar disks and are thus effectively hidden today until bulge-to-disk decompositions are performed. It turns out that there are actually many reasons to favour this scenario.

There is no widely accepted solution as to how the distant objects could have grown into much larger 'spheroids' by today. For instance, major dry mergers can not account for the size evolution; they move galaxies along the mass-size relation rather than off it (e.g. Ciotti \& van Albada 2001; Boylan-Kolchin et al. 2006; see also Bundy et al. 2009; Nipoti et al. 2009; Nair et al. 2011). In addition, based on observations of galaxy pairs, there have been insufficient major (i.e. near equal mass ratio) merger events to explain the removal of every distant, compact massive galaxy (e.g. Man et al. 2015; see also Shih \& Stockton 2011). Similarly, there are not enough satellites observed around massive galaxies for

2 The exceptions are brightest cluster galaxies and luminous $\left(M_{B} \lesssim-20.5 \pm 0.75 \mathrm{mag}\right)$ elliptical galaxies built via major dry merger events, and very faint dwarf elliptical galaxies. minor mergers (e.g. Khochfar \& Burkert 2006; Maller et al. 2006; Naab et al. 2009; Hopkins et al. 2009; McLure et al. 2013) to be the sole explanation (Trujillo 2013, and references therein), and even in $\Lambda \mathrm{CDM}$ simulations there are not enough satellites to have transformed all of the distant spheroids.

It is worth noting that 'normal-sized' (i.e. not compact), massive elliptical-like galaxies are observed at these high-redshifts, co-existing with the 'compact', massive galaxies (e.g. Trujillo et al. 2006b; Mancini et al. 2010; Newman et al. 2010; Bruce et al. 2012; Ferreras et al. 2012; Fan et al. 2013a). Furthermore, new galaxies are known to appear on the "red sequence" (e.g. Bell et al. 2004; Faber et al. 2007; Schawinski et al. 2007, 2014; van Dokkum et al. 2008; Martig et al. 2009; van der Wel et al. 2009; López-Sanjuan et al. 2012; Carollo et al. 2013; Cassata et al. 2013; Keating et al. 2015; Belli et al. 2015). For example, Carollo et al. (2015) detail how past quenching can transform star-forming galaxies and shift them to the early-type sequence. Known as 'progenitor bias', studies which attempt to measure the evolution of the massive quiescent galaxies (e.g. Trujillo et al. 2006b; Ryan et al. 2012; Chang et al. 2013; van der Wel et al. 2014) can suffer from sample confusion because of the emerging population onto the red sequence; such studies are effectively sampling different objects as a function of redshift.

In mid-2011, Graham (2013, see also Driver et al. 2013) suggested that some of the distant objects are likely to be the bulges of today's massive early-type disk galaxies. Indeed, Graham (2013) showed that the distant compact massive objects and massive local bulges occupy the same region in the size-mass and size-density diagram. Dullo \& Graham (2013) additionally showed that they possess the same radial concentration of light, as traced by the Sérsic index (Sérsic 1968, see Graham \& Driver 2005 for a modern review in English). Moreover, massive bulges today are old. Their mass (not to be confused by their luminosity-weighted age) is dominated by old stars (MacArthur et al. 2009) and therefore such luminous bulges should be visible in images of the early universe. That is, some fraction of the compact massive objects reported at $z \sim 2 \pm 0.6$ are expected to be today's compact massive bulges. By calculating the number density of compact massive bulges at $z \sim 0$, done for the first time in this work, we are able to address whether this fraction is low or instead if it might equal 1 and thus fully account for the fate of the distant compact massive objects.

Rather than some great mystery of unexplained galaxy growth, we are exploring the relatively mundane alternative idea that Daddi et al. (2005) and Trujillo et al. (2006a,b) simply observed the bulges of today's bright early-type galaxies at high- $z$ but did not know it. To address this, we have performed a preliminary search for nearby $(\lesssim 100 \mathrm{Mpc})$, early-type disk galaxies with bulges having $R_{\mathrm{e}} \lesssim 2 \mathrm{kpc}$ and $M_{*} \approx 10^{11} M_{\odot}$. This was done by checking on the galaxies presented in a handful of published papers listed in Table [1] This proved to be sufficient to illustrate that compact, massive spheroids exist in substantial numbers around us today. Section 2 of this paper presents the galaxies found, and the structural properties of their spheroidal component are also 
provided there. Section 3 provides a general discussion of these findings and the growth of disks in our Universe. Section 4 finishes by re-iterating our main conclusions.

\section{DATA SAMPLE}

Five survey papers were consulted to check for the existence of compact massive bulges in the nearby Universe. Our criteria was that the stellar mass be greater than $0.7 \times 10^{11} M_{\odot}$ and the major-axis half-light radius be less than $2.5 \mathrm{kp} 3$. The first paper we examined was Dullo \& Graham (2013) which has already broached this topic, and the second was Savorgnan et al. (2015, in preparation) which has carefully modeled the different structural components for 66 galaxies from a sample of 75 (Graham \& Scott 2013; Scott et al. 2013) having directly measured black hole masses. Building on this, we checked two samples dominated by lenticular galaxies. First we inspected the sample of 175 early-type disk galaxies modeled by Laurikainen et al. (2010), and then the ATLAS ${ }^{3 \mathrm{D}}$ sample (Cappellari et al. 2011) of 260 nearby ( $<42 \mathrm{Mpc}$ ), predominantly northern hemisphere, early-type galaxies which have had the stellar bulge-to-total flux ratios derived by Krajnović et al. (2013). The fifth catalog paper that we inspected was the spiral dominated compendium of Graham \& Worley (2008), which resulted in the identification of targets in four additional papers (see Table 1). Having checked these papers, our Table 1 includes 20 known bulges along with the known compact massive galaxy NGC 1277 (van den Bosch et al. 2012), plus one additional galaxy, as opposed to a bulge, which also meets the above size and mass criteira. All but three of the 22 systems have $R_{\mathrm{e}}<2 \mathrm{kpc}$. All but 4 have $M_{*} \geq 0.9 \times 10^{11} M_{\odot}$.

The major-axis, half-light radii of the spheroids are presented in Table 1, based on the galaxy distances which are also listed there. The circularized half-light radii would be even smaller, by a factor of $\sqrt{b / a}$ where $b / a$ is the minor-to-major axis ratio of the bulge. This information was not readily available for many of our bulges, and so we have used the more conservative, larger radii. The published Sérsic indices have additionally been collated and given in Table 1 for ease of reference. Our preference has been for near-infrared data sets because the magnitudes are more reliable: they are less affected by dust and possible low level star formation. The absolute magnitudes were then corrected for redshift dimming $(5 \log [1+z])$, foreground Galactic extinction (Schlafly \& Finkbeiner 2011), and $K$-corrected (1.5z, Poggianti $1997)$ which resulted in insignificant changes of typically 0.01-0.02 mag.

In the case of NGC 5493 (Krajnović et al. 2013), the whole galaxy is rather compact $\left(R_{\mathrm{e}}=2.46 \mathrm{kpc}\right)-$ although this is the largest system in our sample - and massive $\left(M_{*}=10^{11} M_{\odot}\right)$. This galaxy is not unique in the nearby universe. The intermediate-scale disk in NGC 1332, which does not dominate at large radii like large-scale disks do, is such that the size of this galaxy is

\footnotetext{
3 It should be noted that studies of the high' $z$ galaxies typically report the smaller, circularized half-light radii rather than the major-axis half-light radii. Our approach is thus conservative.

${ }^{4}$ Krajnović et al. (2013) reported that their bulge/disk decomposition for NGC 5493 differed markedly from Laurikainen et al. (2010). Given this uncertainty, we use the whole galaxy rather than a result from a decomposition.
}

only slightly larger than $2 \mathrm{kpc}$ (Savorgnan et al. 2015, in preparation). However here we include the bulge rather than the galaxy parameters for NGC 1332. A third and possible fourth example is NGC $1277\left(M_{*}=\right.$ $1.2 \times 10^{11} M_{\odot}$ ) and NGC 5845 (excluded here because $\left.M_{*}=0.5 \times 10^{11} M_{\odot}\right)$, as already pointed out by van den Bosch et al. (2012) and Jiang et al. (2012), respectively. However, if the disks of these galaxies were to continue to grow, until they resembled the more stereotypical S0 galaxies today, with $B / D$ flux ratios of $1 / 3$ and $R_{\mathrm{e} \text {,bulge }} / h_{\text {disk }} \approx 0.2\left(R_{\mathrm{e}, \text { bulge }} / R_{\mathrm{e}, \text { disk }} \approx 0.12\right)$, then their galaxy sizes would likely exceed $2 \mathrm{kpc}$ while the spheroidal component remained compact.

For reference, it was observed that the bulges with stellar masses $M_{*} \gtrsim 2 \times 10^{11} M_{\odot}$ tend to have major-axis, half-light radii $\widetilde{R}_{\mathrm{e}}>2.5 \mathrm{kpc}$, and as such were not included here.

We elected not to apply any internal dust correction to the lenticular galaxies, but only to the spiral galaxies. The most massive lenticular galaxies are not representative of the typical dusty disk galaxy from which the dust corrections stem, and they likely contain little wide-spread dust. The following $K$-band dust correction from Driver et al. (2008) was applied to the bulges of the spiral galaxies.

$$
M_{\text {bulge }}^{\text {corr }}=M_{\text {bulge }}-0.11-0.79[1-\cos (i)]^{2.77},
$$

where $i$ is the inclination of the disk such that $i=90 \mathrm{deg}$ corresponds to an edge-on orientation. For the Dullo \& Graham (2013) sample of five lenticular galaxies, the stellar masses derived from the $V$-band magnitudes (RC3, de Vaucouleurs et al. 1991) agree with those derived from the $K_{s}$-band magnitudes when no dust correction is applied and $M / L_{K_{s}}=0.8$ and $M / L_{V}=2.5$ is used. While the Sombrero lenticular galaxy contains wide-spread dust in its disk, as do other lenticular galaxies (e.g. Temi et al. 2007), it may be that ion sputtering (e.g. Draine 2003) from a hot X-ray gas halo has destroyed the dust in the massive lenticular galaxies, although we have not checked for such X-ray halos in our sample. However, if we are mistaken and dust is present, it will mean that our estimated stellar masses should be increased. As shown in Driver et al. (2008), the average correction due to dust in the thousands of disk galaxies modeled as a part of the Millennium Galaxy Catalog (Liske et al. 2003; Allen et al. 2006) is $<0.14 \mathrm{mag}$ if the inclination is $<45^{\circ}$, and $0.18,0.28$ and $0.45 \mathrm{mag}$ if the inclination is 55,65 and 75 degrees, respectively.

Some of the near-infrared magnitudes in Table 1 were derived from Two Micron All-Sky Survey (2MASS) images (Skrutskie et al. 2006). As noted by Schombert \& Smith (2012), the 2MASS total magnitudes are known to not capture all of a galaxy's light; they miss flux at large radii. However, for most S0s this appears to be contained to, on average, a tenth of a magnitude (Scott et al. 2013, their Figure 2), no doubt due to the rapid decline of the S0 galaxy's outer exponential light profile. For S0s with intermediate (i.e. not large) scale disks that do not dominate their galaxy's light at large radii, and if the Sérsic index of the bulge is large, then several tenths of a mag may be missed, as with the elliptical galaxies. The (Galactic extinction)-corrected $B-K_{s}$ colors (via NED but from the RC3, 2MASS and Schlafly \& Finkbeiner 
TABLE 1

Galaxy Sample.

\begin{tabular}{|c|c|c|c|c|c|c|c|c|c|c|c|}
\hline $\begin{array}{l}\text { Gal. } \\
\text { Id. } \\
(1)\end{array}$ & $\begin{array}{c}\text { Type } \\
(2)\end{array}$ & $\begin{array}{c}\text { Inc. } \\
\text { [deg] } \\
(3)\end{array}$ & $\begin{array}{l}\mathrm{z} \\
(4)\end{array}$ & $\begin{array}{c}\text { Dist } \\
{[\mathrm{Mpc}]} \\
(5)\end{array}$ & $\begin{array}{c}n_{\text {bulge }} \\
(6)\end{array}$ & $\begin{array}{c}R_{\mathrm{e}, \mathrm{bulge}} \\
{[\mathrm{kpc}]} \\
(7)\end{array}$ & $\begin{array}{c}M_{\text {bulge }} \\
{[\mathrm{mag}]} \\
(8)\end{array}$ & $\begin{array}{c}M_{\text {bulge }}^{\text {corr1 }} \\
{[\text { mag] }} \\
(9)\end{array}$ & $\begin{array}{c}M_{\mathrm{bulge}}^{\text {corr } 2} \\
{[\mathrm{mag}]} \\
(10)\end{array}$ & $\begin{array}{l}/ L \\
(11)\end{array}$ & $\begin{array}{c}\text { Mass }_{*, \text { bulge }}^{\text {corr }} \\
{\left[10^{11} M_{\odot}\right]} \\
(12)\end{array}$ \\
\hline \multicolumn{12}{|c|}{ Lenticular galaxies } \\
\hline \multicolumn{12}{|c|}{ Laurikainen et al. (2010, their table 1$) K_{s}$-band bulge magnitude } \\
\hline ESO $337-G 10$ & $\mathrm{SA}(\mathrm{s}) 0^{-}$ & 37.6 & 0.0193 & 79.0 & 1.7 & 1.52 & -24.33 & -24.37 & $\ldots$ & 0.8 & 0.9 \\
\hline NGC 484 & $\mathrm{SAO}^{-}$ & 45.1 & 0.0170 & 68.4 & 2.8 & 1.69 & -24.63 & -24.65 & $\ldots$ & 0.8 & 1.2 \\
\hline NGC 1161 & $\mathrm{SA}(1) 0^{0}$ & 33.6 & 0.0065 & 28.4 & 2.5 & 1.77 & -24.20 & -24.27 & $\ldots$ & 0.8 & 0.8 \\
\hline NGC 3665 & $\mathrm{SA}^{\circ}(\mathrm{s})$ & 39.1 & 0.0069 & 32.4 & 2.7 & 1.98 & -24.36 & -24.37 & $\ldots$ & 0.8 & 0.9 \\
\hline NGC 5266 & $\mathrm{SA}^{-}$ & 54.2 & 0.0100 & 40.8 & 2.8 & 1.84 & -24.79 & -24.82 & $\ldots$ & 0.8 & 1.4 \\
\hline NGC 5419 & $\mathrm{SA}(\mathrm{nl}) 0^{-}$ & 43.9 & 0.0138 & 54.8 & 1.4 & 1.74 & -24.60 & -24.63 & & 0.8 & 1.2 \\
\hline NGC 7796 & so & 27.9 & 0.0112 & 48.5 [Ton] & 2.2 & 1.72 & -24.47 & -24.48 & & 0.8 & 1.0 \\
\hline ATLAS3D: $r^{\prime}$ & band $R_{\mathrm{e}}$ and & n. $B /$ & $/ T \times K_{s}$ & -band galax & y lumin & osity (Capp & ellari et a & 1. 2011 , th & leir table & $3 ; \mathrm{Kr}$ & hović et al. 2013) \\
\hline NGC $4649^{\dagger}$ & So & 39.6 & 0.0037 & 17.3 & 1.8 & $1.42 \pm 0.10$ & -24.22 & -24.22 & & 0.8 & 0.80 \\
\hline NGC 3640 & So & 34.9 & 0.0043 & 26.3 & 2.1 & $2.12 \pm 1.11$ & -24.05 & -24.05 & $\cdots$ & 0.8 & 0.7 \\
\hline NGC 5493* & S0 & 65.2 & 0.0089 & 38.8 & $5.8^{*}$ & $2.46 \pm 0.11^{*}$ & $-24.49^{*}$ & $-24.49^{*}$ & $\ldots$ & 0.8 & $1.0^{*}$ \\
\hline \multicolumn{12}{|c|}{ Savorgnan et al. (2015, in preparation) $3.6 \mu \mathrm{m}$ bulge magnitude } \\
\hline NGC 1316 & $\mathrm{SAB}(\mathrm{s}) 0^{0}$ & 45 & 0.0059 & $20.0[\mathrm{~B} 09]$ & $2.0^{* *}$ & $1.54^{* *}$ & $-25.05^{* *}$ & $-25.05^{* *}$ & $\ldots$ & 0.6 & 1.2 \\
\hline NGC 1332 & $\mathrm{~S}^{-}(\mathrm{s})$ & 73 & 0.0051 & 22.3 [Ton] & $5.3^{* *}$ & $1.95^{* *}$ & $-24.89^{* *}$ & $-24.89^{* *}$ & & 0.6 & 1.1 \\
\hline \multicolumn{12}{|c|}{ Dullo \& Graham (2013) V } \\
\hline NGC 507 & $\mathrm{SA}(\mathrm{r}) 0^{0}$ & 0.0 & 0.0165 & $\begin{array}{r}66.2 \text { [NED] } \\
\text { van }\end{array}$ & $\begin{array}{c}2.2 \\
\text { den Bos }\end{array}$ & $\begin{array}{c}1.71 \\
\text { ch et al. } 2\end{array}$ & $\begin{array}{l}-24.57 \\
012)\end{array}$ & -24.60 & (l) & 0.8 & 1.1 \\
\hline NGC $1277^{*}$ & $\mathrm{~S}^{+}{ }^{+}$pec & 75 & 0.0169 & 73.0 & $2.2^{*}$ & $1.2^{*}$ & 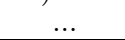 & $\ldots$ & & & $1.2^{*}$ \\
\hline \multirow{2}{*}{\multicolumn{12}{|c|}{$\begin{array}{c}\text { Spiral galaxies } \\
\text { Graham }(2001\end{array}$}} \\
\hline & & \multicolumn{9}{|c|}{ Graham $(2001,2003) K$-band bulge magnitude } & \\
\hline NGC 266 & $\mathrm{SB}(\mathrm{rs}) \mathrm{ab}$ & 27.1 & 0.0155 & $62.8[\mathrm{NED}]$ & 1.4 & 1.25 & -24.14 & -24.17 & -24.28 & 0.8 & 0.9 \\
\hline NGC 2599 & SAa & 29.5 & 0.0158 & $68.4[\mathrm{NED}]$ & 2.1 & 1.71 & -24.45 & -24.47 & -24.58 & 0.8 & 1 \\
\hline NGC 3884 & $\mathrm{SA}(\mathrm{r}) 0 / \mathrm{a}$ & 40.5 & 0.0234 & 103.6 [NED] & 2.3 & 1.64 & -24.41 & -24.43 & -24.55 & 0.8 & 1.1 \\
\hline NGC 7490 & Sbc & 24.5 & 0.0207 & 84.9 [NED] & 2.6 & 1.53 & -23.99 & -24.03 & -24.14 & 0.8 & 0.7 \\
\hline UGC 2862 & $\left(\mathrm{R}^{\prime}\right) \mathrm{SAB}(\mathrm{s}) \mathrm{a}$ & 44.4 & $\begin{array}{r}\text { Sei } \\
0.0224 \\
\text { Möll }\end{array}$ & $\begin{array}{l}\text { igar \& Jame } \\
89.3 \text { [NED] } \\
\text { enhoff \& He }\end{array}$ & $\begin{array}{l}\text { s }(1998) \\
1.9 \\
\text { idt }(200\end{array}$ & $\begin{array}{l}K \text {-band by } \\
1.15 \\
1 \text { 1) } K \text {-band }\end{array}$ & $\begin{array}{c}\text { dlge magn } \\
-24.22 \\
\text { bulge mas }\end{array}$ & $\begin{array}{l}\text { tude } \\
-24.34 \\
\text { nitude }\end{array}$ & -24.48 & 0.8 & 1.0 \\
\hline NGC 3147 & $\mathrm{SA}(\mathrm{rs}) \mathrm{bc}$ & 34.9 & $\begin{array}{l}0.0093 \\
\text { Balcells }\end{array}$ & $\begin{array}{l}43.0[\mathrm{NED}] \\
\text { et al. }(2007 \text {, }\end{array}$ & $\begin{array}{l}3.6 \\
\text { their ta }\end{array}$ & $\begin{array}{l}1.39 \\
\text { ble } 2) K-\mathrm{b}\end{array}$ & $\begin{array}{l}-24.41 \\
\text { and bulge }\end{array}$ & $\begin{array}{l}-24.42 \\
\text { magnitude }\end{array}$ & $\mathrm{e}^{-24.54}$ & 0.8 & 1.1 \\
\hline NGC 6504 & $\mathrm{Sab}$ & $78.5^{\mathrm{D}}$ & $\begin{array}{r}0.0160 \\
\text { Lau }\end{array}$ & rikainen et a & $\begin{array}{l}2.7 \\
\text { l. }(2010\end{array}$ & $\begin{array}{c}1.20 \\
K_{s} \text {-band }\end{array}$ & $\begin{array}{c}-24.11 \\
\text { bulge mag }\end{array}$ & $\begin{array}{l}-24.13 \\
\text { nitude }\end{array}$ & -24.67 & 0.8 & 1.2 \\
\hline NGC 6646 & $\left(\mathrm{R}^{\prime}\right) \mathrm{SAB}(\mathrm{rs}) \mathrm{a}$ & 23.4 & 0.0192 & 81.8 & 2.2 & 2.16 & -24.16 & -24.19 & -24.30 & 0.8 & 0.9 \\
\hline
\end{tabular}

Note. - Column 1: Galaxy identification. * The galaxy rather than bulge parameters are presented. †NGC 4649 (M60) is a particularly difficult galaxy to decompose and as such its bulge parameters may not be reliable. Column 2: Morphological Type. Column 3: Disk inclination such that 90 degrees corresponds to an edge-on disk. Column 4: Redshift taken from the NASA/IPAC Extragalactic Database (NED). Column 5: Distance from corresponding paper unless otherwise specified: NED $=($ Virgo + GA + Shapley $)$ distance from NED using $\left.H_{0}=73 \mathrm{~km} \mathrm{~s}^{-1} \mathrm{Mpc}^{-1}\right)$; B09= Blakeslee et al. $(2009)$; Ton $=$ Tonry et al. (2001) and corrected according to Blakeslee et al. (2002). Column 6: Sérsic index. Column 7: Major-axis, effective half-light radius of the galaxy's bulge component. Column 8: Near-infrared magnitude. Notes: $*=$ galaxy rather than bulge parameters; ** $3.6 \mu \mathrm{m}$ magnitude rather than $K$ - or $K_{s}$-band. Column 9: Magnitude corrected for Galactic dust extinction (Schlafly \& Finkbeiner 2011), $(1+z)^{2}$ cosmological dimming, and $K$-corrected using $+1.5 z$ (Poggianti 1997). Column 10: Magnitude additionally corrected for internal dust using the correction from Driver et al. (2008). Column 11: Stellar mass-to-light ratio (assumes a 12 Gyr old population of solar metallicity and a Chabrier (2003) initial mass function: Baldry et al. 2008, their Figure A1). Column 12: Stellar mass derived using column 9 for the S0s and column 10 for the spirals, together with column 11.

2011) range from about 3.65 to 4.05 . Given the expected color range of 3.85 to 4.03 for a 6 to 12.5 Gyr old population in model S0 galaxies (Buzzoni 2005), perhaps a couple of tenths of mag are missing from some 2MASS magnitudes. If one was to correct for this, it too would act to make the bulge masses bigger than reported here.

A Hubble constant of $H_{0}=73 \mathrm{~km} \mathrm{~s}^{-1} \mathrm{Mpc}^{-1}$ (e.g. WMAP 3-year; Riess et al. 2011) was used. However if it is smaller, for example $67.3 \mathrm{~km} \mathrm{~s}^{-1} \mathrm{Mpc}^{-1}$ (Planck Collaboration 2014), then the absolute magnitudes will brighten by 0.18 mag and the stellar masses will increase by $18 \%$. Based on SNe Ia data, Rigault et al. (2015) have also downwardly revised $H_{0}$ to $70.6 \pm 2.6 \mathrm{~km} \mathrm{~s}^{-1} \mathrm{Mpc}^{-1}$. A mass increase of $18 \%$ would result in 18 of the 22 systems in Table 1 having stellar masses $\geq 10^{11} M_{\odot}$.

\subsection{Example profile: $N G C 5419$}

While information about the surface brightness profile decompositions can be found in the papers mentioned in Table 1, we felt that it may be instructive to include an example of how the outer exponential profile can increase the galaxy size over the bulge size. We have somewhat randomly chosen NGC 5419 from Laurikainen et al. (2010). This was chosen because a) the Laurikainen et al. paper simply contains the greatest number of compact massive spheroids in our Table 1, and b) this particular galaxy was modelled by Dullo \& Graham (2014) as an elliptical galaxy while they noted that a disk might be present. Sandage \& Tammann (1981) did not support the original elliptical galaxy classification in the Second Reference Catalogue of de Vaucouleurs et al. (1976), but 


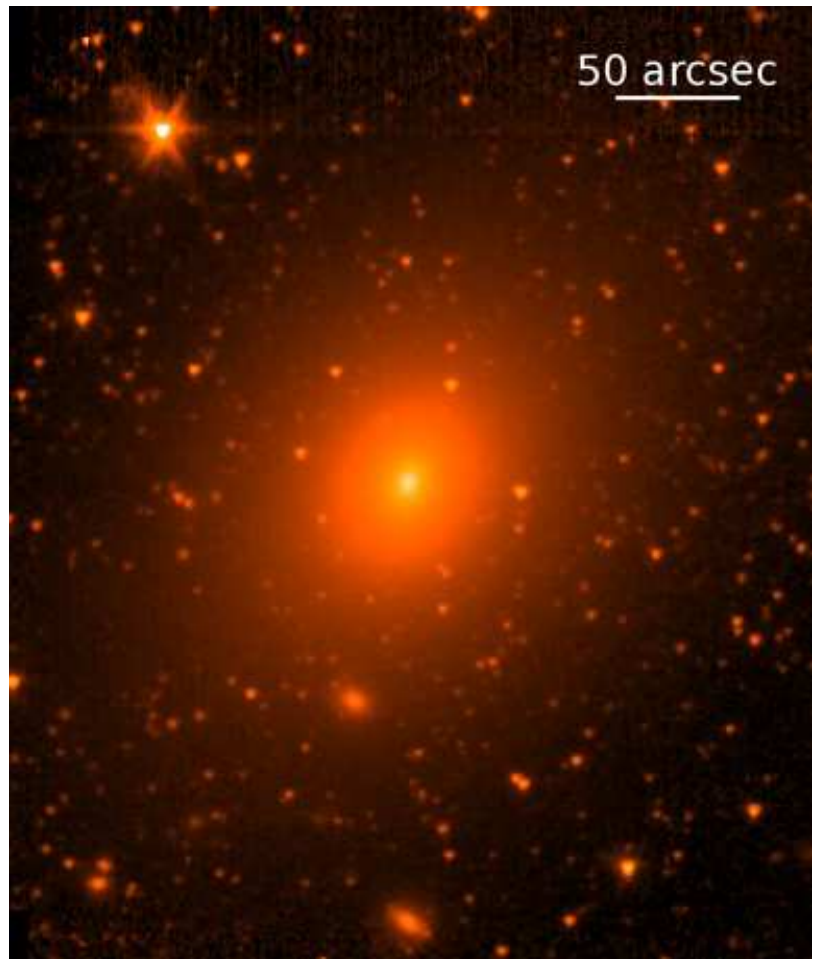

FIG. 1.- Spitzer Space Telescope 3.6 $\mu \mathrm{m}$ image of NGC 5419.

instead considered NGC 5419 to be a lenticular galaxy, as did Laurikainen et al. (2010) based on their near-infrared $K$-band analysis.

Therefore, we re-investigate this galaxy using a Spitzer Space Telescope $3.6 \mu \mathrm{m}$ image (see Figure 1), which was reduced following the procedures described in Savorgnan et al. (2015, in prep.). The light profile was extracted by allowing the ellipticity and position angle to vary about a fixed center using the IRAF task ELLIPSE (Jedrzejewski et al. 1987) and is shown in Figure 2

Our modelling of the $3.6 \mu \mathrm{m}$ light profile within $60^{\prime \prime}$ matches the solution to the $K$-band data in Laurikainen et al. (2010). We also find that a single Sérsic function is inadequate; the curvature of the light profile, and the residual light profile, reveal the presence of additional components. Figure 2 shows how the addition of an outer exponential, and a very faint lens (fit with a Ferrers function), provides a better fit. These were the components employed by Laurikainen et al. (2010). From this, one can see that the half-light radius of the galaxy $\left(R_{\mathrm{e}, \mathrm{gal}}=53^{\prime \prime} .6\right)$ is much greater than the half light radius of the inner spheroid $\left(R_{\mathrm{e}, \mathrm{sph}}=8^{\prime \prime} .4\right)$. Laurikainen et al. (2010) reported that their bulge+lens+disk model had a bulge Sérsic index $n=1.4$ and half light radius $R_{\mathrm{e}}=6^{\prime \prime} .5$. They reported a disk scale length $h=32^{\prime \prime} .3$, while our exponential model has $h=33^{\prime \prime} .1$.

One may wonder if there is also a contribution from an extended envelope around this galaxy due its location at the centre of the poor cluster Abell S753. Indeed, Sandage \& Bedke (1994) referred to this galaxy as having an extended outer envelope, and Seigar et al. (2007, see also Pierini et al. 2008) established that intracluster light tends to have an exponential profile, i.e. the same radial decline as seen in disks. However, Bettoni et al. (2001) have reported a rotational velocity reaching $90 \mathrm{~km} \mathrm{~s}^{-1}$ by

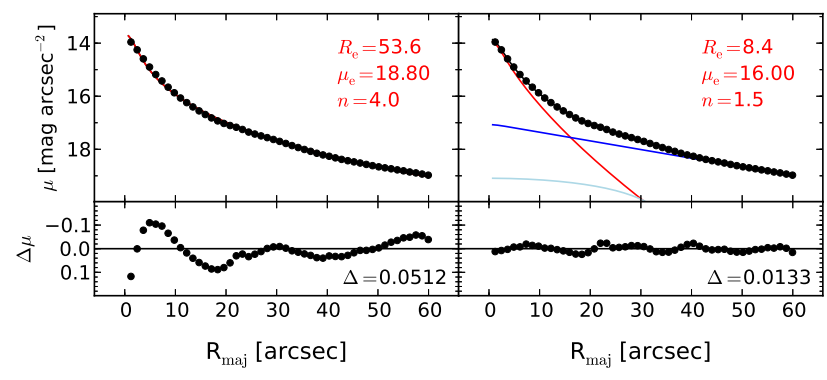

Fig. 2.- Left panel: Single Sérsic fit to the $3.6 \mu \mathrm{m}$, major-axis light profile of NGC 5419. Right panel: As in Laurikainen et al. (2010), a Sérsic bulge (red), plus exponential function (dark blue), plus Ferrers function for a lens (cyan) has been fit. The root mean square of the deviations of the surface brightness data, $\mu$, about the model is given by $\Delta$.

the inner $5^{\prime \prime}$, betraying the presence of at least an inner disk. In an extreme scenario, one may speculate that this galaxy has an intermediate-scale disk (the 'lens' component in Figure 2, which shows up in both the ellipticity and position angle profiles) plus intracluster stars that produce the near-exponential light profile at larger radii. More extended kinematics would be helpful in discriminating between these options.

The cluster-centric location of NGC 5419 is interesting, and it further prompted us to pursue the suggestion by L.Cortese (2015, priv. comm.) to check on the environment (e.g. field, group, cluster) of our sample. Table 2 shows this information, along with the $R_{e} / h$ size and $B / T$ flux ratios obtained by the papers given in Table 1 . While a few of the galaxies are the brightest of their small galaxy group, NGC 1316 (the interacting galaxy Fornax A) is the only other member of a substantialsized group. This rules out the idea that the compact massive spheroids might be encased in an exponentiallike $3 \mathrm{D}$ envelope of intracluster light rather than a $2 \mathrm{D}$ disk.

\section{RESULTS}

\subsection{The mass-size and size-concentration diagrams}

In Figure 3a we do not compare our data with the sizemass relation for early-type galaxies as given by Shen et al. (2003) because of the biases in their data which are explained in Graham \& Worley (2008). Lange et al. (2015) have however fit the double power-law model from Shen et al. (2003) to the galaxy size-mass data from several thousand nearby $(0.01 \leq z \leq 0.1)$ earlytype (morphologically-identified diskless) galaxies taken from the Galaxy And Mass Assembly (GAMA) survey (Driver et al. 2011). The galaxy magnitudes had been converted into stellar masses by Taylor et al. (2011), and the effective half light galaxy sizes derived by Kelvin et al. (2012) who fit single Sérsic models to images in ten bands $\left(u g r i z Z Y J H K_{s}\right)$. We have taken the $K_{s}$-band re- 
TABLE 2

Galaxy PROPERTIES.

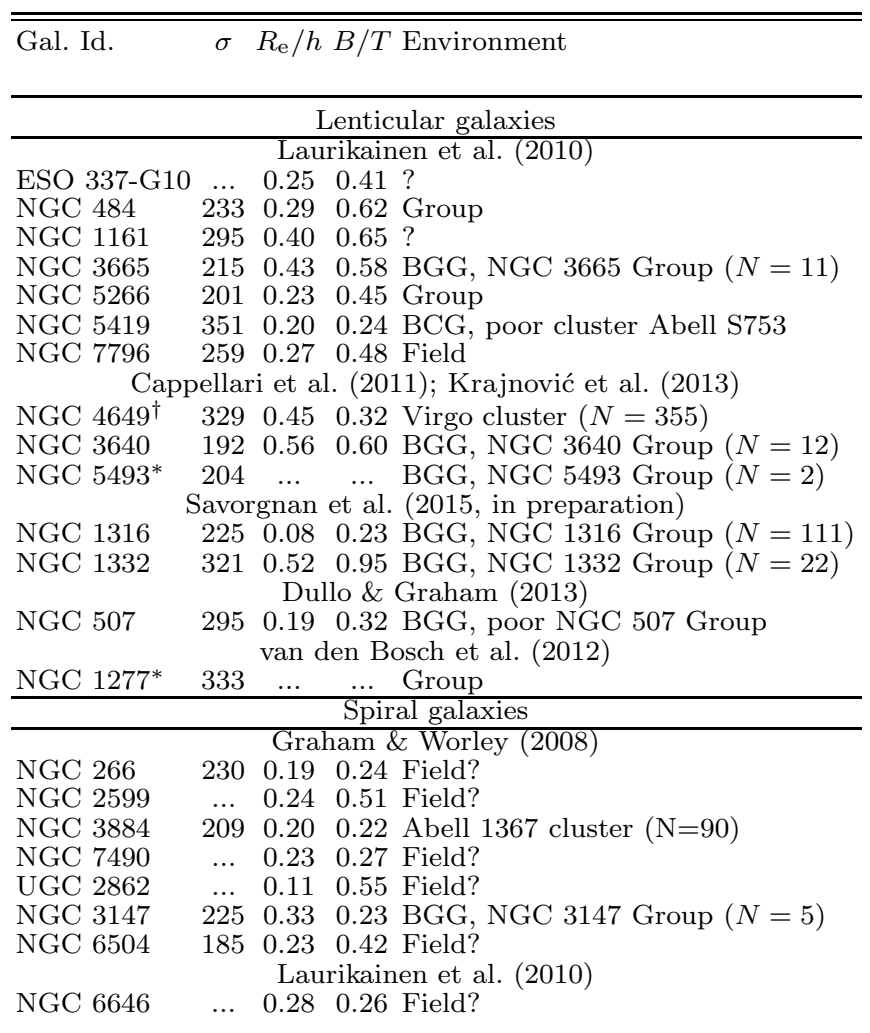

Note. - Column 1: Galaxy identification. * The galaxy rather than bulge parameters were used. †Bulge/disk parameters may not be reliable. $¥ N G C 1332$ has had its disk fit with a Sérsic model having $n=0.5$, for which the scale length $h=R_{\text {e,disk }} / 0.82$ (rather than $R_{\mathrm{e}, \text { disk }} / 1.68$ as is the case when $n=1$ ). Column 2: Velocity dispersion $\left(\mathrm{km} \mathrm{s}^{-1}\right.$ ) from HyperLeda (Makarov et al. 2014), except for NGC 1277 (van den Bosh et al. 2012). Columns 3 and 4: Bulgeto-disk size ratio and bulge-to-total flux ratio, respectively. For the lenticular galaxies (and NGC 6646), values were taken from the respective papers. For the spiral galaxies, the dust-corrected values were derived in Graham \& Worley (2008). Column 5: Environment of the galaxy. $\mathrm{BCG}=$ Brightest Cluster Galaxy. BGG $=$ Brightest Group Galaxy. $N$ represents the number of galaxies with known radial velocities in the group (Makarov \& Karachentsev 2011; Tully et al. 2013). Field? = No group known to authors, and the galaxy appears to be in the field from looking at Digitized Sky Survey images available via NED.

sults from Lange et al. (2015) and show their mass-size relation in Figure 3 a. Given that Figure 6 from Lange et al. (2015) reveals that the double power-law cannot fully capture the curvature in the GAMA data at $M_{*}>1$ $2 \times 10^{11} M_{\odot}$ - where galaxies have larger radii than the double power-law model — we have therefore additionally included the mass-size relation from Graham et al. (2006, see Eq.2.11 in Graham 2013). While Figure 3a reveals that the mass-size relation of local early-type galaxies have larger radii at a given mass than the distant spheroids, it also reveals that the masses and sizes of our local bulges overlap with those of the distant spheroids (Damjanov et al. 2011). Our data appears more clustered in Figure 3 a simply because we have been stricter with the mass and size limit for our local bulge sample.

In Figure 3b we show the sizes and Sérsic indices of our local compact massive systems, and the overlap with galaxies in the redshift interval $1.4<z<2.7$, as given by Damjanov et al. (2011, their Table 2). We have excluded from Damjanov et al. those galaxies with no reported Sérsic index and those fit with an $R^{1 / n}$ surface brightness model having a fixed value of $n$, such as 4 . As with our bulge data, the high- $z$ galaxy data in Damjanov et al. has also been taken from a compilation of different surveys: 17 in their case. Their galaxy selection criteria is thus varied, and it can be seen in Figure 3 to contain a much larger range of sizes and masses than our data. Comparing the data points, we conclude that not all compact, massive spheroids need to have undergone significant structural and size evolution since $z \lesssim 2.5$.

\subsection{The number density of local, compact massive spheroids}

Twenty one of the 22 systems (20 bulges plus 2 galaxies) are within $90 \mathrm{Mpc}$, with the additional system at 103.6 Mpc. Excluding this furthest bulge gives a number density of $6.9 \pm 1.5 \times 10^{-6} \mathrm{Mpc}^{-3}$ for the sample of 21. All of these systems have stellar masses in the range $0.7 \times 10^{11}<M_{*} / M_{\odot}<1.4 \times 10^{11}$. Ten of the 13 systems with $M_{*} \geq 10^{11} M_{\odot}$ are within $70 \mathrm{Mpc}$, giving a similar number density of $7.0 \pm 2.2 \times 10^{-6} \mathrm{Mpc}^{-3}$. It needs to be remembered that these densities are a lower limit because we have not conducted a volume-limited, all-sky survey for compact massive spheroids. However, the ATLAS ${ }^{3 D}$ survey did sample all of the bright galaxies over half the sky to a depth of $42 \mathrm{Mpc}$. Given that it contains three compact, massive systems, this corresponds to a number density of $(1.9 \pm 1.1) \times 10^{-5} \mathrm{Mpc}^{-3}$. This value is $2.75( \pm 58 \%)$ times higher, but there is a larger uncertainty assuming Poissonian errors.

While Taylor et al. (2010) reported a reduction, at fixed size and mass, of at least 5000 in the co-moving number density of compact massive galaxies from $z \sim 2.3$ to $z \sim 0.1$, we find that there is a roughly comparable (within a factor of a few) number density of compact massive systems at $z=0$ and $z \sim 2.5$. Bezanson et al. (2009) report a number density of $(3 \pm 1) \times 10^{-5} \mathrm{Mpc}^{-3}$ at this higher redshift, for systems with stellar mass densities greater than one billion solar masses within their innermost sphere of radius $1 \mathrm{kpc}$ (or $M_{*}>10^{11} M_{\odot}$, $\left.R_{\mathrm{e}}<2.88 \mathrm{kpc}\right)$. Muzzin et al. (2013, their Figure 5) report a number density of $\sim 5 \times 10^{-5} \mathrm{Mpc}^{-3}$ at $2<z<3$ for all (compact and extended) quiescent galaxies with $M_{*} \sim 10^{11} M_{\odot}$, in fair agreement with the value of $\sim 4 \times 10^{-5} \mathrm{Mpc}^{-3}$ for the quiescent galaxies at $z=3 \pm 0.5$ with $M_{*}>0.4 \times 10^{11} M_{\odot}$ reported by Straatman et al. (2014). At these high redshifts, it is speculated here that the bulk of the disk formation (which will remove "galaxies" from satisfying the compactness criteria), may be yet to occur and thus one may have a cleaner sample of 'naked-bulges' with which to make a comparison with the number density of bulges in the local universe. At yet higher redshifts the 'naked-bulges' may likely still be developing themselves (e.g. Dekel \& Burkert 2014).

Barro et al. (2013) report a similar number density as

\footnotetext{
5 The usual dependence on the Hubble constant - in this case $h_{73}^{3}$ - has been omitted given that the spherical volumes used have not been exactly matched to any particular outer-most galaxy's distance.
} 


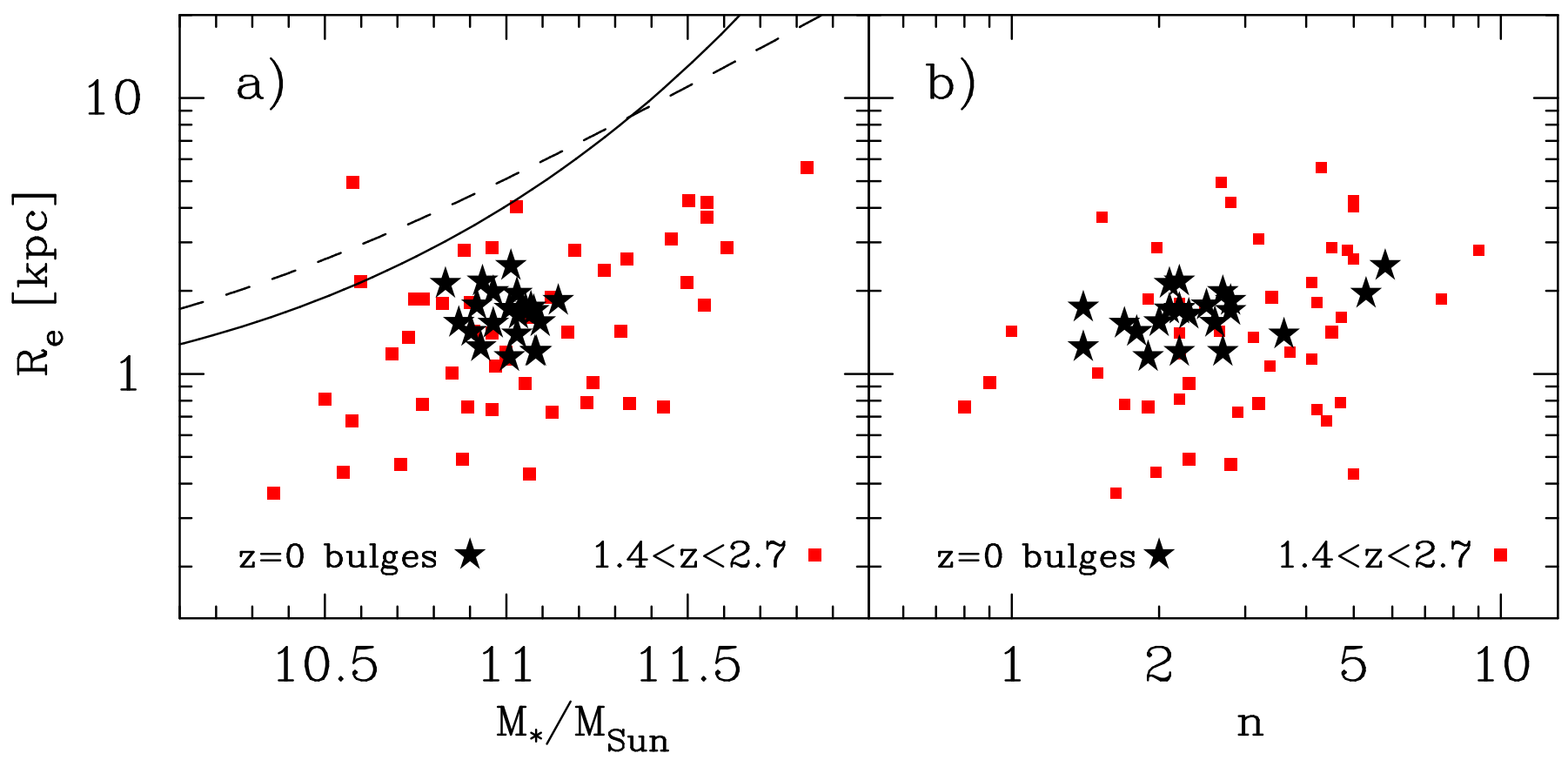

FIG. 3.- Panel a) Stellar mass-size diagram, where the sizes of the local bulges are represented here by the major-axis effective half light radius $R_{\mathrm{e}}$. The curves show the parameterized fit to local "elliptical" galaxy data from Graham et al. (2006, solid line) and Lange et al. (2015, specifically their Figure A9j and Eq.3, and their final $K$-band entry in Table 3, dashed line). The $z=0$ bulge data is from Table! 1 while the $1.4<z<2.7$ data is from Damjanov et al. (2011). Local bulges with $M_{*} \gtrsim 2 \times 10^{11} M_{\odot}$ have $R_{\mathrm{e}} \geq 2.5 \mathrm{kpc}$ and were thus not included here because they are not considered to be compact galaxies. Panel b) Size-concentration diagram, where the concentration is quantified by the Sérsic index $n$.

Bezanson et al. (2009) at $z=2.5$, even though Barro et al. used a lower stellar mass limit of $0.1 \times 10^{11} M_{\odot}$ for their sample (cf. $1.0 \times 10^{11} M_{\odot}$ used by Bezanson et al. 2009). Barro et al. reported that their number density increased as the redshift dropped, peaking at about $2.3 \times 10^{-4}$ $\mathrm{Mpc}^{-3}$ by $z=1.2$, before dropping to lower densities as the redshift decreased further. Taken together, this suggests that the most massive, quiescent spheroids were in place first, with the less massive spheroids appearing later at lower redshifts (possibly connecting with the luminous blue compact galaxies forming at $z<1.4$, Guzmán et al. 1997). The above peak density at $z=1.2$ is, at least in part, larger than the value we have found because Barro et al. included galaxies having a much broader range in stellar mass than we did. In a separate study, van der Wel et al. (2014) used a stellar mass limit $>0.5 \times 10^{11} M_{\odot}$ and found a peak density at $z \sim 1.2$ of $1 \times 10^{-4} \mathrm{Mpc}^{-3}$.

The mass range sampled in our study only spans a factor of two, from 0.7 to $1.4 \times 10^{11} M_{\odot}$. Our number density per unit dex in mass, as recorded in mass functions, is therefore five times higher, giving $3.5 \times 10^{-5} \mathrm{Mpc}^{-3}$ $\mathrm{dex}^{-1}$ (or $\approx 10^{-4} \mathrm{Mpc}^{-3} \mathrm{dex}^{-1}$ if using the volumelimited ATLAS ${ }^{3 \mathrm{D}}$ results) at $M_{*} \approx 10^{11} M_{\odot}$. These values are roughly 2-6 times lower than that from Barro et al. (2013), whose galaxy mass range exceeded 1 dex, and roughly 1-3 times lower than that from van der Wel et al. (2014), whose mass range was less than 1 dex. A proper comparison is however complicated because the local bulge mass function is not quite flat from 0.1 to $1.4 \times 10^{11} M_{\odot}$, but increases as one moves to the lowermass end (e.g. Driver et al. 2007). For this reason, our number density for local compact massive bulges, as estimated above, is expected to be less than the actual number density in the decade-wide mass range from say 0.14 to $1.4 \times 10^{11} M_{\odot}$. Using a constant stellar $M / L$ ratio across this mass range, to convert the bulge luminosity function in Driver et al. (2007) into a mass function, the actual number density in this decade range might be $\sim 40 \%$ higher, although predicting this properly requires knowledge of the slope of the mass function for local compact bulges. We hope to perform a more complete, volume-limited investigation of compact massive bulges within $100 \mathrm{Mpc}$ in a forthcoming paper, enabling us to construct the mass function of local compact bulges with $M_{*}>10^{10} M_{\odot}$. This can then be better compared with the mass function at higher redshifts.

The WIde-field Nearby Galaxy-cluster Survey (WINGS) has reported the existence of many compact massive galaxies in nearby $(0.04<z<0.07)$ clusters. Including substantially lower mass galaxies than us, Valentinuzzi et al. (2010) reported that $22 \%$ of the WINGS galaxies with $0.3 \times 10^{11}<M_{*} / M_{\odot}<4 \times 10^{11}$ are compact, with a median size of $1.61 \pm 0.29 \mathrm{kpc}$ (and a median Sérsic index of $3 \pm 0.6$ ). For their cluster galaxy sample they derived a lower limit (because they excluded field galaxies) for the number density of $(1.31 \pm 0.09) \times 10^{-5} \mathrm{Mpc}^{-3}$ within the co-moving volume between $z=0.04$ and $z=0.07$. This density drops to $(0.46 \pm 0.05) \times 10^{-5} \mathrm{Mpc}^{-3}$ when they increase their lower mass limit from $0.3 \times 10^{11} M_{\odot}$ to $0.8 \times 10^{11} M_{\odot}$ (their Table 1). They observed that the bulk of their compact galaxies are lenticular galaxies, which are known to have $\sim 30 \%$ smaller disk sizes in galaxy cluster environments (e.g. Gutiérrez et al. 2004; Head et al. 2014).

Using the Padova Millennium Galaxy and Group Catalogue (PM2GC) spanning $0.03<z<0.11$, Poggianti et 
Graham et al.

al. (2013a,b) explored outside of clusters - most galaxies reside in the field or group environment - and concluded that $4.4 \%$ of local field and group galaxies with stellar masses $0.3 \times 10^{11}<M_{*} / M_{\odot}<4 \times 10^{11}$ are compact, and that this $4.4 \%$ - most of which are lenticular galaxies - has a median mass-weighted age of $9.2 \mathrm{Gyr}$, and corresponds to a number density of $(4.3) \times 10^{-4} \mathrm{Mpc}^{-3}$, or basically $3.2 \times 10^{-4} \mathrm{Mpc}^{-3} \mathrm{dex}^{-1}$ given their mass range sampled.

Although the above two studies treated the lenticular galaxies as single component systems when measuring both their ages and their circularized half-light radii, we suspect that it is the bulges of these galaxies which are the primary, compact massive spheroidal component. In a sense, we may therefore be dealing with the same type of galaxy as they are. While we do not try and resolve the question as to why their compact, massive galaxies were not found by others who searched in the SDSS database, we do provide a couple of comments. Disk galaxies viewed edge-on will of course have circularized half-light radii which are notably smaller than the values obtained if they are viewed face-on. It may be of interest to see if the Valentinuzzi et al. (2010) and Poggianti et al. (2013a,b) detections are dominated by massive, edge-on disk galaxies. It may additionally be interesting to see the results (sizes, masses, number densities) after careful bulge/disk decompositions have been performed for these WINGS and PM2GC samples.

To recap our findings, we measure a lower limit of $3.5 \times 10^{-5} \mathrm{Mpc}^{-3} \mathrm{dex}^{-1}$ (or $\approx 10^{-4} \mathrm{Mpc}^{-3} \mathrm{dex}^{-1}$ if using the volume-limited ATLAS ${ }^{3 \mathrm{D}}$ results) for compact $\left(R_{\mathrm{e}} \lesssim 2 \mathrm{kpc}\right)$ bulges with $M_{*} \approx 10^{11} M_{\odot}$.

\section{DISCUSSION}

\subsection{The rise of disks}

As noted in the Introduction, within the literature, minor mergers are the overwhelmingly preferred, albeit still problematic, solution to try and transform the compact, massive spheroids at high redshifts into larger spheroids by today. While our discovery of numerous compact massive spheroids at $z=0$ suggests that this evolutionary path did not always transpire, we can still ask about minor mergers. If (wet or dry) minor mergers had built a large fraction of each disk around today's compact massive spheroids, then it may be telling us that there is something not quite right with $\Lambda \mathrm{CDM}$ simulation\$6. This is because the simulations contain somewhat random orientations of satellite galaxies which would not result in the formation of a disk, although see Tempel et al. (2015). However if minor mergers have built the disks, the observations may then be telling us that most central galaxies have had preferred 'Great Planes' on which their minor neighbors were located prior to infall and disk (rather than bulge) building. Some support for this idea can be found in the disks-of-satellites around the Milky Way and Andromeda (Kroupa et al. 2005; Metz et al. 2007; Ibata et al. 2013; Pawlowski et al. 2014). What is of course different in our scenario is that these Great Planes are not just a recent, local, phenomenon but would need to have always been present over the

\footnotetext{
${ }^{6}$ Combes et al. (2015a) provides an interesting review of simulations with MOND in regard to bulge formation.
}

past 10-13 Gyrs (see Goerdt, Burkert \& Ceverino 2013).

Hammer et al. $(2005,2009)$ have reasoned that many rotating stellar disks could have been built in major gasrich mergers, with the disk forming due to the net angular momentum of the gas in the merger event (e.g. Barnes 2002; Robertson et al. 2006). Even in gas-poor (dry) mergers, Naab \& Burkert (2003) report that disks can form if the net angular momentum is not canceled out (Fall 1979). Given that rotating spiral galaxies at $z \approx 0.65$ are half as abundant as they are today (Neichel et al. 2008), disk growth since $z=2 \pm 0.6$ has obviously occurred, and the outer regions of galaxies with prominant bulges are known to be younger (e.g. Pérez et al. 2013; Li et al. 2015). Arnold et al. (2011) additionally remarked that lenticular galaxies might form through a two-phase inside-out assembly with the inner regions built early via a violent major merger, and wet minor mergers (rather than gas accretion) subsequently contributing to their outer parts.

It is recognized that there has not been enough galaxy merger events to transform the distant compact massive galaxies into today's ordinary-sized elliptical galaxies (e.g. Man et al. 2015), and therefore the above mechanisms cannot, on their own, be invoked to fully explain the growth of disks around the compact massive galaxies seen at high- $z$ such that they have become today's lenticular galaxies with typical disk-to-bulge flux ratios of 3 (i.e. bulge/total $=1 / 4$ ). Observing higher gas fractions of lower metallicity at higher redshifts, Béthermin et al. (2015) favour large gas reservoirs over major mergers as the cause of the intense star formation observed in massive galaxies at high redshifts. Following the idea of cyclical galaxy metamorphosis (White \& Rees 1978; White \& Frenk 1991; Navarro \& Benz 1991; Steinmetz \& Navarro 2002; see also Bournard \& Combes 2002), Graham (2013) advocated earlier suggestions that 'cold' gas flows may have contributed to the development of these disks, either via spherical accretion of $\sim 10^{4}-10^{5} \mathrm{~K}$ gas (e.g. Birnboim \& Dekel 2003; Birnboim et al. 2007) or streams (e.g. Kereš et al. 2005; Dekel et al. 2009; Kereš \& Hernquist 2009; Danovich et al. 2014).

This alternative process, which can operate in parallel with mergers (e.g. Welker et al. 2015), involves the accretion of gas from not just the halo (e.g. Kauffmann et al. 1993, their section 2.7; 1999, their section 4.5; Genzel et al. 2006), and possible molecular gas reservoirs for some galaxies (e.g. Tacconi et al. 2008; Santini et al. 2014; Chapman et al. 2015), but also cold filamentary flows from the cosmic web (e.g. Ceverino et al. 2010, 2012; Goerdt et al. 2012; Rubin et al. 2012; Stewart et al. 2013). Although such cold gas accretion is yet to be convincingly demonstrated as a common phenomenon, it has the power to subsequently build a disk that transforms into stars. Indeed, it has been noted for well over a decade that the mass of a galaxy could double, due to gas accretion, in just a few Gyr (e.g. Katz et al. 1996; Bournaud \& Combes 2002). The discovery of very low metallicity gas (0.02 solar) $37 \mathrm{kpc}$ from a sub- $L_{*}$ galaxy with solar metallicity by Ribaudo et al. (2011) revealed the presence of such gas, and we now know that there is lots of low metallicity gas in the halos of galaxies (e.g. Lehner et al. 2013; Prochaska et al. 2014). Furthermore, Bouché et al. (2013) have revealed the inflow (as opposed to just presence) of this material around a $z=2.3$ galaxy 
that has kinematics, metallicity and star formation typical of a rotationally supported disk, and it is such that the gas accretion rate is well-matched to the star formation rate (see also Conselice et al. 2013). It has also been established that there is a high spatial covering fraction of Lyman- $\alpha$ gas clouds within $300 \mathrm{kpc}$ of all galaxy types at high- $z$ (Wakker \& Savage 2009; Prochaska et al. 2011; Thom et al. 2011; Stocke et al. 2013; Tumlinson et al. 2013), and a review of cool gas in high $z$ galaxies can be found in Carilli \& Walter (2013). In particular, even quiescent elliptical galaxies can have a massive cool reservoir around them (Thom et al. 2012; Tumlinson et al. 2013; Zhu et al. 2014; O'Sullivan et al. 2015). Although not surrounding a pre-existing compact, massive bulge, Prescott et al. (2015) report on the rotation of an $80 \mathrm{kpc}$ Lyman- $\alpha$ nebula at $z \sim 1.67$ with an implied total mass of $3 \times 10^{11} M_{\odot}$ within a $20 \mathrm{kpc}$ radius. While this is a developing field, extensive evidence for gas accretion at many redshifts is reviewed by Combes $(2014,2015 b)$.

It seems likely that the compact massive galaxies would act as natural gravitational seeds around which filaments or streams of cold gas would flow inward and build disks that form stars. The existence of early-type galaxies with dual, large-scale counter-rotating stellar disks (e.g. NGC 4550, Rubin et al. 1992; NGC 3032, Young et al. 2008) supports the idea of disk growth via the accretion of external gas clouds (e.g. Coccato et al. 2014, and references therein), although it may also be due to minor mergers. Presumably feeding is usually from the same direction, given the low frequency of significant (by mass) counter-rotation, and after settling to the mid-plane the rotation is aligned. Small counter-rotating stellar disks and kinematically decoupled cores do however reveal that this is not always the case, and gas accretion can form interesting features such as warps, gas-star misalignments and polar rings (e.g. Briggs 1990; Jog \& Combes 2009; Davis et al. 2011; Mapelli et al. 2015). However rather than only random accretion orientations, Pichon et al. (2011) explains how cold streams can build a disk in a coherent planer manner (see also Danovich et al. 2012, 2014; Prieto et al. 2013; Stewart et al. 2013; Cen 2014; Wang et al. 2014), which is of key importance. A second key aspect is the rapid formation of some of these disks (e.g. Agertz et al. 2009; Brooks et al. 2009) which would naturally explain the older ages of many lenticular galaxy disks today. It also implies significant galaxy growth independent of the merging of distinct entities such as dark matter halos. This process of gas accretion is still observed today in massive early-type galaxies (e.g. Davis et al. 2011).

Disk growth in early-type galaxies is an on-going phenomenon (e.g. Yi et al. 2005; Kaviraj et al. 2007; Fabricius et al. 2014), albeit at lower levels today as less gas is available (e.g. Combes et al. 2007; Sage et al. 2007; Huang et al. 2012; Catinella et al. 2013). The molecular gas usually resides in kpc-scale, rotating disks (e.g. Inoue et al. 1996; Wiklind et al. 1997; Okuda et al. 2005; Das et al. 2005), as does the HI gas (e.g. Serra 2012, 2014, and references therein). Young et al. (2008), for example, detail the slow ongoing growth of the disk in NGC 4526, a galaxy whose bulge has a half light radius equal to $1.43 \mathrm{kpc}$ (Krajnović et al. 2013) and a stellar mas: 7 of

7 Based on $M_{r^{\prime}}=-20.4$ mag and using $M / L_{r^{\prime}}=5$ (Krajnović
$0.55 \times 10^{11}$ (which, along with other bulges, was not massive enough to be included in our sample). Perseus A is yet another example which is also still accreting cold gas (Salome et al. 2006).

As noted in Section 2, NGC 1277, NGC 1332, NGC 5493 and NGC 5845 are examples of local earlytype galaxies where the disk does not dominate the light at large radii, as in proto-typical lenticular galaxies. These disks are an order of magnitude larger than nuclear disks, and referred to as intermediate-sized disks. Given that the amount of gas accretion can vary, it would be natural for such disks to exist. They are not a new phenomenon (e.g. Scorza \& van den Bosch 1998, and references therein), but some readers may not be familiar with their existence. At $z \approx 2$, the lower mass spheroids, with less gravitational pull, and which likely formed from a smaller over-density in the early universe, may naturally experience a smaller subsequent supply of gas from cold streams and build their disks more gradually. Freefloating, "compact elliptical" dwarf galaxies (e.g. Huxor et al. 2013; Paudel et al. 2014) might represent spheroids which never acquired a significant disk, while those in the vicinity of much larger neighbours are thought to have been largely stripped of their disks.

The theoretical work of Steinmetz \& Navarro (2002, and references therein) suggested that a galaxy's morphology is a transient phenomenon. Galaxies do not simply progress from the "blue cloud" to the "red sequence" (Faber et al. 2007) but can move in the opposite direction. While the latter pathway may not be traversed in full today, because less gas is available, there are fledged examples of galaxies in the 'green valley' (e.g. Cortese \& Hughes 2009; Marino et al. 2011). Elliptical galaxies may initially be built through major mergers of disk galaxies (and perhaps from a violent disk instability, Ceverino et al. 2015), and then proceed to grow a new stellar disk through gas accretion (White \& Frenk 1991, p.77), which remains intact until the next significant merger (see Salim et al. 2012 and Conselice et al. 2013 for supporting arguments, and Fang et al. 2012 and Bresolin 2013 for caution in some cases). The number of cycles may however be low (i.e. 1 or 2) rather than several (3 to 5). Evidence for a bulge-then-disk scenario may exist within the Milky Way (e.g. Zoccali et al. 2006). Furthermore, the hierarchical models from Khochfar \& Silk (2006), for example, present diskless galaxies at $z=2$ which evolve into disk galaxies with a bulge-to-total mass ratio equal to 0.2 by $z=0$ due to gas accretion from the halo, cold streams, and minor mergers.

There have recently been reports of infant, premature disks detected in some of the high-redshift, compact massive galaxies (Chevance et al. 2012, and references therein). In a study of 14 compact massive galaxies at $z=2.0 \pm 0.5$, van der Wel et al. (2011) reported that $65( \pm 15) \%$ are disk-dominated, appearing highly flattened on the sky and having disks with a median half-light radius of $2.5 \mathrm{kpc}$. This corresponds to a median scale length of $1.5 \mathrm{kpc}$, which is half the size of disks today, e.g. Graham \& Worley (2008, their Figure 3). These high- $z$ disks were observed to harbor compact central components, i.e. bulges. Although most early-type galaxies around us today are known to be lenticular disk 
galaxies - thanks to studies such as ATLAS3D ${ }^{3 \mathrm{D}}$ (Emsellem et al. 2011) — there are of course still some massive, slow or non-rotating, elliptical galaxies. Some of these may be the 'ordinary-sized' elliptical galaxies, observed at high- $z$, while others likely formed from more recent, major merger events. Some of the 'ordinary-sized' elliptical galaxies at high- $z$ may have also acquired disks, but they would appear as intermediate-sized disks if insufficient gas was accreted to build a larger scale disk around an already large galaxy.

Following the deep observations by Szomoru et al. (2010) to verify the compactness of a galaxy at $z=1.91$ found by Daddi et al. (2005), we note that future work should be mindful that shallow surveys at any redshift could miss the outer disks of galaxies to varying degrees and may largely just recover the inner bulge if they are particularly shallow. If that was to occur, one would effectively, although accidentally, manage to identify higher number densities of compact massive systems.

\subsection{Stellar Ages, and stellar mass loss}

The above scenario for disk growth around compact "bulges" requires the massive bulges of nearby disk galaxies to be old. Although beyond the scope of the current investigation, it will be of interest to explore the ages of the bulges and disks in the current sample of galaxies. However MacArthur, González \& Courteau (2009) have already shown that bulges in both early- and late-type disk galaxies do indeed have old mass-weighted ages, with less than 25 per cent by mass of the stars being young, second or third generation stars built from metal enriched gas. Based on stellar populations and radial gradients, MacArthur et al. (see also Fisher et al. 1996) concluded that early-formation processes are common to bulges and that secular processes or 'rejuvenated' star formation generally contributes minimally to the stellar mass budget of bulges (see also Moorthy \& Holtzman 2006; Thomas \& Davies 2006; and Jablonka et al. 2007), yet it has biased luminosity-weighted age estimates in the past. Such 'frostings' of young stars, of up to 25 per cent by mass, can give the impression of positive luminosity-weighted age gradients, i.e. bulges are younger at their centres, and have misled some studies (which assumed a single stellar population) into missing the fact that the bulk of the stellar mass in most bulges is old 9 . This follows Kuntschner \& Davies (1998) who revealed that the obvious lenticular galaxies in the Fornax cluster have ages which are younger than the more spheroid-dominated galaxies in the cluster.

These works imply that the bulk of the stellar mass in today's massive bulges already existed at high redshifts, and therefore these stellar systems should be what

\footnotetext{
8 If so, one may need a mechanism to curtail disk growth, such as more efficient AGN feedback due to their lower stellar density, or hot X-ray halos.

9 A caveat is that disks are built both around and within 'bulges' The surface mass density of disks is higher in their centers than their outskirts. Therefore, disks which are relatively younger than their bulges will contribute younger stars where the bulge resides, but the dominant stellar population will be old at the center of the system. It is of course also known that when disks do form, they may generate bars which can in turn further build on the "bulge" via secular processes. At the same time, strong bars can stall the inward accretion of gas, restricting it to the outer parts of the disk until the bar weakens (Bournaud \& Combes 2002).
}

we are observing in deep images of our young Universe. Curiously, Saracco et al. (2009) reported that there are two kinds of early-type galaxy at $z \approx 1.5$ : an old $(\sim 3.5$ Gyr) population which needs to experience a factor of 2.5-3 size evolution to have sizes equal to today's earlytype galaxies, and a young ( $\sim 1 \mathrm{Gyr})$ population (perhaps those which have recently acquired their disks) which already have sizes consistent with today's population of early-type galaxies. In this regard, it will be interesting to know if their old population is best described with a single Sérsic model, while their young population is better described by a bulge+disk model rather than a singlecomponent Sérsic model. Furthermore, if a younger disk has formed, or bulges developed at different epochs, they may have different initial stellar mass functions (IMFs), resulting in differing composite IMFs for the early-type galaxies today (e.g. Dutton et al. 2013, McDermid 2015, and references therein).

Gradual stellar mass loss due to stellar winds is a part of the ageing process for passively evolving spheroids (e.g. de Jager et al. 1988; Ciotti et al. 1991; Jungwiert et al. 2001), as is the conversion of visible stars into dark remnants such as neutron stars and stellar mass black holes. If a fraction $x$ of the initial mass is lost from a galactic system due to stellar winds, or Type Ia supernovae clearing out gas, then there will be an adiabatic expansion because the galaxy is no longer as tightly bound and it will therefore reach a new equilibrium such that its size has increased by $1 /(1-x)$ (e.g. Jeans 1961; Hills 1980 , their eq.6). Of course if any stellar ejecta remains in a galaxy - which seems likely in massive galaxies with strong potential wells - , perhaps eventually ending up as hot X-ray gas, then the expansion of the galaxy will be reduced depending on the radial expanse of this gas. After the young stars $(<1 \mathrm{Gyr})$ have evolved, stellar winds likely account for just a $\lesssim 10 \%$ reduction to the stellar mass of the passive, compact massive galaxies seen at $z \sim 2$ (Damjanov et al. 2009, but see Poggianti et al. 2013b). Fan et al. (2008) had previously suggested that AGN feedback may blow out the gas in distant massive galaxies and cause them to expand by factors of 3 or more. Of course if this had happened, then we would be left having to explain where all of the compact massive bulges in the Universe today came from.

\subsection{Stellar density}

Given the smaller half-light radii that early-type galaxies had at higher redshifts, before they acquired their disks, the stellar densities within those half-light radii were 1 to 2 orders of magnitude greater than the stellar densities inside the half-light radii of today's large early-type galaxies. This led Zirm et al. (2007) to conclude that it is a problem for models of early-type galaxy formation and evolution. Buitrago et al. (2008) claimed that within the inner $1 \mathrm{kpc}$ of the high- $z$ galaxies, they have densities equal to globular clusters. This led them to advocate a scenario in which globular clusters and distant compact massive galaxies may have a similar origin, while at the same time suggesting that the more massive halos (not the globular clusters) started collapsing earlier and dragged along a larger amount of baryonic matter that later formed stars. However they overestimated the typical globular cluster size by a factor of $10 / 3$, and thus under-estimated a typical globular cluster's density by a 
factor of 37, undermining their conclusions.

Bezanson et al. (2009) showed that the inner regions $(<1 \mathrm{kpc})$ of today's early-type galaxies have stellar densities which are 2-3 times lower than those of the distant compact massive galaxies of the same mass. However, the majority of galaxies in our sample have bulge-tototal ratios of $1 / 2-1 / 4$ (Table 2), while Laurikainen et al. (2010) found an average ratio of $1 / 4$ for their sample of lenticular galaxies. That is, the local massive bulges are 2-4 times less massive than the galaxy within which they reside. In comparing galaxies of the same mass in Figure 2d from Bezanson et al. (2009), one is effectively comparing the high redshift galaxies with local bulges that are 2-4 times less massive, possibly accounting for the lower density observed in these lower-mass bulges. If one compares the quiescent, compact massive galaxies at high-redshift with today's bulges of the same mass, one may find that they have the same density within the inner kpc.

Hopkins et al. (2009) reported that there is no central $(<1 \mathrm{kpc}$ ) density mismatch (see also van Dokkum et al. 2014). They suggested that "the entire population of compact, high-redshift red galaxies may be the progenitors of the high-density cores of present-day ellipticals", by which they mean spheroids. We however consider the evolution to have been very different, such that a $2 \mathrm{D}$ disk is built within and around the spheroid that undergoes no substantial size-evolution, rather than the continual development of a 3D envelope around a spheroid that effectively undergoes significant size growth.

\subsection{Velocity Dispersions}

If there is indeed no evolution of the distant spheroids, then their velocity dispersion should remain the same. Now, if a stellar disk builds within and around them, then the galaxy mass today will have increased. As a result, if one was to compare the velocity dispersions of the distant compact spheroids with the velocity dispersions of $z=0$ disk galaxies having the same total stellar mass, one would actually be sampling local bulges which are less massive than the distant spheroids. The local galaxies of the same mass, containing compact bulges of 2-4× lower mass, are naturally expected to have lower velocity dispersions. Indeed, this general behavior has been observed (e.g. Cenarro \& Trujillo 2009; Cappellari et al. 2009; Newman et al. 2010).

If one assumes $M_{*} \propto \sigma^{4}$, then a $2-4 \times$ difference in stellar mass will be associated with a $\sim 1.2-1.4 \times$ difference in velocity dispersion. If one attempts to bypass the photometric stellar mass estimate and use a dynamical / virial mass estimate $\left(\sigma^{2} R_{\mathrm{e}, \text { gal }}\right)$ for the lenticular galaxies, the same issue occurs. This is because of the enhanced

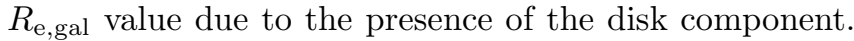
If a galaxy's half-light radius is 3-6 times larger than the half-light radius of its central bulge, then the velocity dispersion of the bulge coupled with the galaxy size will produce a galaxy virial mass which is 3-6 times larger than what one would obtain for the bulge. This will act to artificially separate the compact high- $z$ spheroids from local massive bulges in a diagram of dynamical mass versus velocity dispersion. A note to keep in mind is that disk growth, as with envelope growth, may lead to the compression of the original inner bulge and a slight en- hancement in the central velocity dispersion (Andredakis 1998; Debattista et al. 2013).

\subsection{Depleted cores}

The presence of partially depleted cores in massive spheroids is thought to be a result of major, dry merger events (e.g. Begelman et al. 1980; Faber et al. 1997; Merritt et al. 2007). The large elliptical galaxies with sizes several times that of the $\operatorname{high}^{6} z$, compact massive spheroids, and with a deficit of stars over their inner tens of parsecs to a few hundred parsecs, likely formed this way. The orbital decay of the binary supermassive black hole, created by the galaxy merger, proceeds by slingshotting the central stars out of the core of the newly formed galaxy. However, as noted by Dullo \& Graham (2013), the presence of such partially depleted cores in the bulges of massive disk galaxies (e.g. Dullo \& Graham 2013) presents a conundrum because these disk galaxies may be unlikely to have formed from such major merger event 10 . One potential solution is that the bulges may have formed first, and the disks were subsequently accreted and grew (Graham 2013; Driver et al. 2013). It may therefore be interesting to check for partially depleted cores in the current sample of disk galaxies in Table 1. Application of the core-Sérsic model (Graham et al. 2003) to Hubble Space Telescope images - due to their superior spatial resolution over a sufficiently wide field of view - will reveal which bulges have an inner deficit of flux relative to the inward extrapolation of their outer Sérsic light profile. Knowledge of which bulges have partially depleted cores, coupled with information about their stellar age, should shed even further light on the formation history.

\subsection{Formation paths and a cautionary remark on the $n=2$ or $n=2.5$ division of bulges}

Surveying predominantly compact, passive, spheroidal galaxies at $1.4<z<2.0$, with $10^{10}<M_{*} / M_{\odot}<10^{11}$, Cimatti et al. (2008, see van Dokkum et al. 2008 for higher redshifts and higher masses) noted that the galaxy sizes are typically such that $R_{\mathrm{e}} \lesssim 1 \mathrm{kpc}$, and are thus much smaller than early-type galaxies of comparable mass in the present-day Universe. However, the bulges of most disk galaxies at $z \approx 0$ have compact sizes with $R_{\mathrm{e}} \lesssim 2 \mathrm{kpc}$ and many bulges with $10^{10}<M_{*} / M_{\odot}<10^{11}$ have $R_{\mathrm{e}} \lesssim 1 \mathrm{kpc}$ (e.g. Graham \& Worley 2008; Graham 2013, his Figure 1). The growth of disks in and around the compact, high- $z$ spheroids could therefore explain their apparent disappearance by today.

This possible explanation may at first glance appear somewhat at odds with the claim in the interesting paper by van Dokkum et al. (2013) that disk models, in which bulges were fully assembled at high redshift and disks gradually formed around them, can be ruled out. However the first distinction in these two remarks is of course that disks do not simply form around spheroids but are additionally embedded within them; which is why bulge/disk decompositions continue the disk all the way into the centers of galaxies. It would therefore be of interest to perform a bulge/disk decomposition of the aver-

10 Major galaxy collisions may be more inclined to result in elliptical galaxies than disk galaxies, although see Naab \& Burkert (2003). 
age surface density profiles, at different redshift intervals, that were constructed by van Dokkum et al. (2013, their Figure 3 ). This would allow one to check how well their $z=0$ (Milky Way)-like galaxy profile that they built resembles the Milky Way, and thus to know if their stacked profiles are reliable or perhaps effected by dust or some other issue 11]. A reliable decomposition of an evolving population (free from 'progenitor bias') would of course enable one to quantify how much the disk and bulge (including pseudobulge) components have changed between the different redshifts. The second point of distinction is that van Dokkum et al. (2013) were referring to the inner $2 \mathrm{kpc}$ radius of (Milky Way)-mass spiral galaxies, rather than $10^{11} M_{\odot}$ mass galaxies. The Milky Way has a bulge mass of $0.91 \times 10^{10} M_{\odot}$ and a bulge-to-total stellar mass ratio of 0.15 (Licquia et al. 2014); it also has a half-light radius of $0.66 \mathrm{kpc}$ (Graham \& Driver 2007). Therefore, within an inner radius of $2 \mathrm{kpc}$, the mass of (Milky Way)like galaxies are dominated by the disk rather than the bulge. Due to the greater prominence of bulges in earlytype disk galaxies than in late-type disk galaxies (see Figure 21 in Graham 2001), the inner regions of today's massive galaxies - which host the once distant, compact massive spheroids - are expected to have changed less over time, as already observed by van Dokkum et al. (2010, their Figure 6).

Within the bulge-then-disk growth scenario, the existence of the distant massive spheroids having $n<2$ implies that local galaxies with bulges having $n<2$ need not be pseudobulges formed from the secular evolution of a stellar disk. This topic is reviewed in Graham (2013, 2014) where it is noted that bulges with Sérsic $n<2$ need not be pseudobulges even if they are also rotating (e.g. Eliche-Moral et al. 2011; Scannapieco et al. 2011; Keselman \& Nusser 2012; Saha et al. 2012; dos Anjos \& da Silva 2013; Graham 2014; Querejeta et al. 2015).

In passing we remark that formation paths in galaxy clusters in which the disks of spiral galaxies fade and lose their pattern, and possibly end up with relatively brighter bulges (e.g. Johnston et al. 2012, and references therein), to become lenticular galaxies can still operate. That is, lenticular galaxies likely have more than one evolutionary path, as can their bulges (e.g. Cole et al. 2000; Springel et al. 2005), and the presence of both classical and pseudobulges in the same lenticular galaxy would seem to support this (e.g. Erwin et al. 2003). At the same time, the preservation, i.e. the lack of evolution, of compact massive spheroids from $z=2 \pm 0.6$ until today has implications for studies of the evolution of the (black hole)-bulge mass scaling relations (reviewed in Graham 2015 , and references therein). The $M_{\mathrm{bh}} / M_{\text {bulge }}$ ratio would not be expected to decrease in these particular systems since approx 1.4 .

McLure et al. (2013) reported that $44 \pm 12 \%$ of their $z=1.4 \pm 0.1$ sample of relatively passive galaxies (specific star formation rate, $\mathrm{sSFR} \leq 0.1 \mathrm{Gyr}^{-1}$ ) with $M_{*} \geq$ $0.6 \times 10^{11} M_{\odot}$ have a disk-like morphology. While at face

\footnotetext{
11 The lack of any central bulge within the inner kpc of the $z=0$ profile is at odds with (Milky Way)-like disk galaxies, and the high average Sérsic index $(n=2.6 \pm 0.4)$ also suggests that their average profile is not representative of (Milky Way)-like galaxies, but rather an intermediate-luminosity early-type galaxy which have a very different bulge-to-total mass ratio.
}

value this claim may appear to support the notion that flattened 2D disks have developed, their morphological (disk-like) claim was based on a Sérsic index divide at $n=2.5$ such that they labeled galaxies with $n<2.5$ to be late-type galaxies. They adopted this divide because Shen et al. (2003) had used it to separate the bright, local galaxy population. Shen et al. (2003) could do this because their $S D S S$ sample contained relatively few dwarf early-type galaxies which have $n<2.5$. However bulges can have $n<2.5$. Therefore, some/many(?) of the galaxies in the sample of McLure et al. (2013) with $n<2.5$ may still be rather "naked bulges", rather than disk-dominated galaxies. Indeed, the similar distributions for their passive sample with $n<2.5$ and $n \geq 2.5$ in the size-mass diagram (their Figure 8) would suggest this.

The practice of considering all high- $z$, compact, massive objects to be disk galaxies if their Sérsic index is less than 2-2.5 is unfortunately rather common (e.g. Hathi et al. 2008; Fathi et al. 2012; Chevance et al. 2012; Muzzin et al. 2012) and may have effectively mis-led many researchers. For this reason we have provided this cautionary text against this practice.

\section{CONCLUSIONS}

The compact massive galaxies at redshifts $z \approx 2 \pm 0.6$ have similar stellar masses and sizes (and thus stellar densities), and radial concentrations of light, as bright bulges in local disk galaxies. Moreover, the number density is not different by hundreds or thousands but is within a factor of a few. We have identified 21 compact $\left(R_{\mathrm{e}} \lesssim 2 \mathrm{kpc}\right)$, massive $\left(0.7 \times 10^{11}<M_{*} / M_{\odot}<1.4 \times 10^{11}\right)$ spheroids within $90 \mathrm{Mpc}$, giving a number density of $6.9 \times 10^{-6} \mathrm{Mpc}^{-3}$ (or $3.5 \times 10^{-5} \mathrm{Mpc}^{-3}$ per unit dex in stellar mass). This is however a lower limit because we have not performed a volume-limited search. Based on a sub-sample taken from a smaller volume-limited sample, this density may be $2.75( \pm 58 \%)$ times higher at around $\times 10^{-4} \mathrm{Mpc}^{-3} \mathrm{dex}^{-1}$.

This observation eliminates the need to grow all of the high- $z$ compact massive spheroids by a factor of 3 to 6 by $z=0$, a challenge which had remained unexplained for the past decade. Rather, many of these high$z$ spheroids need to remain largely unchanged in order to match the massive bulges in today's early-type disk galaxies. Therefore, any study which may have claimed to have accounted for the size growth of quiescent galaxies would have inadvertently, and most likely unknowingly, introduced an equally puzzling problem. If all the distant, compact massive spheroids had evolved, we would then be faced with a second unexplained mystery, namely, where did the compact massive bulges in the local universe come from and why are they not observed in deep images of the $z=2.0 \pm 0.6$ universe.

We conclude that stellar disks have since grown around many compact massive spheroids observed at $z=2.0 \pm$ 0.6 and in so doing transformed their morphological type from elliptical to early-type disk galaxy (i.e. lenticular galaxies and early-type spiral galaxies). Following Graham (2013), we again speculate that some of the less massive compact spheroids at high-redshifts may now reside at the centres of late-type spiral galaxies, and/or are today's compact dwarf elliptical galaxies - which were either largely stripped of their disk or never acquired one. 
The authors thank Francoise Combes, Luca Cortese, Karl Glazebrook and Barbara Catinella for kindly reading and providing helpful comments on this manuscript. A.G. thanks the organizers and participants of the enlightening conference "The Role of Hydrogen in the Evolution of Galaxies" 15-19 September 2014, Kuching, Malaysia (Borneo). This research was supported by the Australian Research Council through funding grant
FT110100263.

This work is based on observations made with the IRAC instrument (Fazio et al. 2004) on board the Spitzer Space Telescope, which is operated by the Jet Propulsion Laboratory, California Institute of Technology under a contract with NASA. This research made use of the NASA/IPAC Extragalactic Database (NED: http://ned.ipac.caltech.edu) and the HyperLeda database (http://leda.univ-lyon1.fr).

\section{REFERENCES}

Agertz, O., Teyssier, R., \& Moore, B. 2009, MNRAS, 397, L64 Allen, P., Driver, S. P., Graham, A. W., Cameron, E., Liske, J., Cross, N. J. G., \& De Propris, R. 2006, MNRAS, 371, 2

Andredakis, Y. C. 1998, MNRAS, 295, 725

Arnold, J. A., Romanowsky, A. J., Brodie, J. P., et al. 2011, ApJ, 736, LL26

Balcells, M., Graham, A. W., \& Peletier, R. F. 2007, ApJ, 665, 1104

Baldry, I. K., Glazebrook, K., \& Driver, S. P. 2008, MNRAS, 388, 945

Barnes, J. E. 2002, MNRAS, 333, 481

Barro, G., Faber, S. M., Pérez-González, P. G., et al. 2013, ApJ, 765,104

Begelman, M.C., Blandford, R.D., \& Rees, M.J. 1980, Nature, 287, 307

Bell, E. F., Wolf, C., Meisenheimer, K., et al. 2004, ApJ, 608, 752

Belli, S., Newman, A.B., Ellis, R.S. 2015, ApJ, 799, 206

Béthermin, M., Daddi, E., Magdis, G., et al. 2015, A\&A, 573, A113

Bettoni, D., Falomo, R., Fasano, G., et al. 2001, A\&A, 380, 471

Bezanson, R., van Dokkum, P. G., Tal, T., et al. 2009, ApJ, 697, 1290

Birnboim, Y., \& Dekel, A. 2003, MNRAS, 345, 349

Birnboim, Y.. Dekel, A., \& Neistein, E. 2007, MNRAS, 380, 339

Blakeslee, J. P., Lucey, J. R., Tonry, J. L., et al. 2002, MNRAS, 330,443

Blakeslee, J. P., Jordán, A., Mei, S., et al. 2009, ApJ, 694, 556 [B09]

Bouché, N., Murphy, M. T., Kacprzak, G. G., et al. 2013, Science, 341,50

Bournaud, F., \& Combes, F. 2002, A\&A, 392, 83

Boylan-Kolchin, M., Ma, C.-P., \& Quataert, E. 2006, MNRAS, 369,1081

Bresolin, F. 2013, ApJ, 772, L23

Briggs, F. 1990, ApJ, 352, 15

Brooks, A. M., Governato, F., Quinn, T., Brook, C. B., \& Wadsley, J. 2009, ApJ, 694, 396

Bruce V.A., Dunlop J.S., Cirasuolo M., et al. 2012, MNRAS, 427, 1666

Buitrago F., Trujillo I., Conselice C.J., et al. 2008, ApJ, 687, L61

Bundy, K.. Fukugita, M., Ellis, R. S., et al. 2009, ApJ, 697, 1369

Buzzoni, A. 2005, MNRAS, 361, 725

Capaccioli, M. 1987, in IAU Symp. 127, Structure and Dynamics of Elliptical Galaxies (Dordrecht: Reidel), 47

Capaccioli, M. 1990, in Bulges of Galaxies, European Southern Observatory Conference and Workshop Proceedings, 35, 231

Cappellari, M., di Serego Alighieri, S., Cimatti, A., et al. 2009, ApJ, 704, L34

Cappellari, M., Emsellem, E., Krajnović, D., et al. 2011, MNRAS, 413, 813

Carrasco E.R., Conselice C.J., Trujillo I. 2010, MNRAS, 405, 2253

Carilli, C. L., \& Walter, F. 2013, ARA\&A, 51, 105

Carollo, C. M., Bschorr, T. J., Renzini, A., et al. 2013, ApJ, 773,

Carollo, C. M., Cibinel, A., Lilly, S. J., et al. 2015, ApJ, submitted (arXiv:1402.1172

Carter, D. 1987, ApJ, 312, 514

Cassata, P., Giavalisco, M., Williams, C. C., et al. 2013, ApJ, 775,106

Catinella, B., Schiminovich, D., Cortese, L., et al. 2013, MNRAS, 436, 34

Cen, R. 2014, ApJ, 789, L21

Cenarro, A. J., \& Trujillo, I. 2009, ApJ, 696, 43

Ceverino, D., Dekel, A., \& Bournaud, F. 2010, MNRAS, 404, 2151

Ceverino, D., Dekel, A., Mandelker, N., et al. 2012, MNRAS, 420 3490

Ceverino, D., Dekel, A., Tweed, D., \& Primack, J. 2015, MNRAS, 447, 3291

Chabrier G., 2003, PASP, 115, 763

Chang, Y.-Y., van der Wel, A., Rix, H.-W., et al. 2013, ApJ, 762 83
Chapman, S.C., Bertoldi, F., Smail, I., et al. 2015, MNRAS in press (arXiv:1501..02839)

Chevance, M., Weijmans, A.-M., Damjanov, I., et al. 2012, ApJ, $754, \mathrm{~L} 24$

Cimatti, A., Cassata, P., Pozzetti, L., et al. 2008, A\&A, 482, 21

Cimatti A., Nipoti C., Cassata P. 2012, MNRAS, 422, L62

Ciotti, L., D'Ercole, A., Pellegrini, S., \& Renzini, A. 1991, ApJ, 376,380

Ciotti L., \& van Albada T. S. 2001, ApJ, 552, L13

Coccato, L., Morelli, L. Pizzella, A., Corsini, E.M., Bontà, E.D., Fabricius, M. 2015, IAU Symp. 309, eds. B.L. Ziegler, F. Combes, H. Dannerbauer, M. Verdugo, p.133

Cole, S., Lacey, C. G., Baugh, C. M., \& Frenk, C. S. 2000, MNRAS, 319, 168

Combes, F., Young, L. M., \& Bureau, M. 2007, MNRAS, 377, 1795

Combes, F. 2014, Structure and Dynamics of Disk Galaxies, Edited by M.S. Seigar and P. Treuthardt. ASP Conference Series, 480,211

Combes, F. 2015a, in "Galactic Bulges", E. Laurikainen, D. Gadotti, R. Peletier (eds.), Springer

Combes, F. 2015b, in Lessons from the Local Group, ed. K. C. Freeman, B. G. Elmegreen, D. L. Block, and M. Woolway, Dordrecht: Springer (arXiv:1405.6405)

Conselice, C. J., Mortlock, A., Bluck, A. F. L., Grützbauch, R., \& Duncan, K. 2013, MNRAS, 430, 1051

Cortese, L., \& Hughes, T. M. 2009, MNRAS, 400, 1225

Daddi, E., Renzini, A., Pirzkal, N., et al. 2005. ApJ, 626, 680

Damjanov, I., McCarthy, P. J., Abraham, R. G., et al. 2009, ApJ, 695,101

Damjanov, I., Abraham, R. G., Glazebrook, K., et al. 2011, ApJ, 739, L44

Damjanov, I., Hwang, H. S., Geller, M. J., \& Chilingarian, I. 2014, ApJ, 793, 39

Danovich, M., Dekel, A., Hahn, O., \& Teyssier, R. 2012, MNRAS, 422,1732

Danovich, M., Dekel, A., Hahn, O., Ceverino, D., \& Primack, J. 2014, MNRAS, submitted (arXiv:1407.7129)

Das, M., Vogel, S. N., Verdoes Kleijn, G. A., ODea, C. P., \& Baum, S. A. 2005, ApJ, 629, 757

Davis, T. A., Alatalo, K., Sarzi, M., et al. 2011, MNRAS, 417, 882

Debattista, V. P., Kazantzidis, S., \& van den Bosch, F. C. 2013 , ApJ, 765, 23

De, T., Chattopadhyay, T., \& Chattopadhyay, A. K. 2014, PASA, 31,47

De Jager, C., Nieuwenhuijzen, H., \& Van der Hucht, K. A. 1988 A\&AS, 72,259

Dekel, A., Birnboim, Y., Engel, G., et al. 2009, Nature, 457, 451

Dekel, A., \& Burkert, A. 2014, MNRAS, 438, 1870

de Vaucouleurs, G., de Vaucouleurs, A., \& Corwin, J. R. 1976 Second reference catalogue of bright galaxies, Austin: University of Texas Press

de Vaucouleurs, G., de Vaucouleurs, A., Corwin, H. G., Jr., Buta, R. J., Paturel, G., Fouque, P. 1991, Third Reference Catalog (Berlin/ Heidelberg/New York: Springer)

D'Onofrio, M., Zaggia, S. R., Longo, G., Caon, N., \& Capaccioli, M. 1995, A\&A, 296, 319

dos Anjos S., \& da Silva, M. B. 2013, Memorie della Societa Astronomica Italiana Supplementi, 25, 33

Draine, B.T. 2003, ARA\&A, 41, 241

Driver, S. P., Allen, P. D., Liske, J., \& Graham, A. W. 2007, ApJ, 657, L85

Driver, S. P., Popescu, C. C., Tuffs, R. J., et al. 2008, ApJ, 678, L101

Driver, S. P., Hill, D. T., Kelvin, L. S., et al. 2011, MNRAS, 413, 971

Driver, S. P., Robotham, A. S. G., Bland-Hawthorn, J., et al. 2013, MNRAS, 430, 2622

Dullo, B. T., \& Graham, A. W. 2013, ApJ, 768, 36

Dullo, B. T., \& Graham, A. W. 2014, MNRAS, 444, 2700

Dutton, A. A.., Treu, T., Brewer, B. J., et al. 2013, MNRAS, 428, 3183 
Eliche-Moral, M. C., González-García, A. C., Balcells, M., et al. 2011, A\&A, 533, 104

Emsellem, E., Cappellari, M., Krajnović, D., et al. 2011, MNRAS, 414, 888

Erwin, P., Beltrán, J. C. V., Graham, A. W., \& Beckman, J. E. 2003, ApJ, 597, 929

Faber, S. M., Tremaine, S., Ajhar, E. A., et al. 1997, AJ, 114, 1771

Faber, S. M., Willmer, C. N. A., Wolf, C., et al. 2007, ApJ, 665, 265

Fabricius, M. H., Coccato, L., Bender, R., et al. 2014, MNRAS, 441, 2212

Fall, S. M. 1979, Nature, 281, 200

Fan, L., Lapi, A., De Zotti, G., \& Danese, L. 2008, ApJ, 689, L101

Fan, L., Chen, Y., Er, X., et al. 2013a, MNRAS, 431, L15

Fan, L., Fang, G., Chen, Y., et al. 2013b, ApJ, 771, L40

Fang, J. J., Faber, S. M., Salim, S., Graves, G. J., \& Rich, R. M. 2012, ApJ, 761, 23

Fang, G.-W., Ma, Z.-Y., Chen, Y., Kong, X. 2015, Research in Astronomy and Astrophysics, in press (arXiv:1501.01107)

Fathi, K., Gatchell, M., Hatziminaoglou, E., \& Epinat, B. 2012, MNRAS, 423, L112

Fazio, G. G., Hora, J. L., Allen, L. E., et al. 2004, ApJS, 154, 10

Ferreras I., Pasquali A., Khochfar S., et al. 2012, AJ, 144, 47

Fisher D., Franx M., Illingworth G. 1996, ApJ, 459, 110

Genzel, R., Tacconi, L. J., Eisenhauer, F., et al. 2006, Nature, 442,786

Goerdt, T., Dekel, A., Sternberg, A., Gnat, O., \& Ceverino, D. 2012, MNRAS, 424, 2292

Goerdt, T., Burkert, A., \& Ceverino, D. 2013, (arXiv:1307.2102)

Forero-Romero, J.E., González, R. 2015, ApJ, 799, 45

Graham, A. W. 2001, AJ, 121, 820

—. 2003, AJ, 125, 3398

-. 2013, in "Planets, Stars and Stellar Systems", Volume 6, p.91-140, T.D.Oswalt \& W.C.Keel (Eds.), Springer Publishing (arXiv:1108.0997)

2014, in Structure and Dynamics of Disk Galaxies, Edited by M.S. Seigar and P. Treuthardt. ASP Conference Series, 480, 185 - 2015, in "Galactic Bulges", E. Laurikainen, R.F. Peletier \& D. Gadotti (Eds.), Springer Publishing (arXiv:1501.02937)

Graham, A. W., Colless, M. M., Busarello, G., Zaggia, S., \& Longo, G. 1998, A\&AS, 133, 325

Graham, A. W.. \& Driver, S. P. 2005, PASA, 22, 118

Graham, A. W., \& Driver, S. P. 2007, ApJ, 655, 77

Graham, A. W., Erwin, P., Trujillo, I., \& Asensio Ramos, A. 2003, AJ, 125, 2951

Graham A. W., Merritt D., Moore B., Diemand J., Terzić B., 2006, ApJ, 132, 2711

Graham, A. W., \& Scott, N. 2013, ApJ, 764, 151

Graham, A. W., \& Worley, C. C. 2008, MNRAS, 388, 1708

Gutiérrez, C. M., Trujillo, I., Aguerri, J. A. L., Graham, A. W., \& Caon, N. 2004, ApJ, 602, 664

Guzmán, R., Gallego, J., Koo, D. C., Phillips, A. C., Lowenthal, J. D., Faber, S. M., Illingworth, G. D., \& Vogt, N. P. 1997, ApJ, 489, 559

Hammer, F., Flores, H., Elbaz, D., et al. 2005, A\&A, 430, 115

Hammer, F., Flores, H., Puech, M., et al. 2009, A\&A, 507, 1313

Hathi, N. P., Jansen, R. A., Windhorst, R. A., et al. 2008, AJ, 135,156

Head,' J., Lucey, J., Hudson, M., Smith, R. 2014, MNRAS, 440, 1690

Hills, J. G. 1980, ApJ, 235, 986

Hopkins, P. F., Bundy, K., Murray, N., et al. 2009, MNRAS, 398, 898

Huang, S., Haynes, M. P., Giovanelli, R., \& Brinchmann, J. 2012, ApJ, 756, 113

Huxor, A. P., Phillipps, S., \& Price, J. 2013, MNRAS, 430, 1956

Ibata, R. A., Lewis, G. F., Conn, A. R., et al. 2013, Nature, 493, 62

Inoue, M. Y., Kameno, S., Kawabe, R., \& Inoue, M. 1996, AJ, 111,1852

Jablonka P., Gorgas J., Goudfrooij P. 2007, A\&A, 474, 763

Jeans, J.H. 1961, Astronomy and Cosmogony (New York: Dover), p. 299

Jedrzejewski, R. I. 1987, MNRAS, 226, 747

Jiang, F., van Dokkum, P., Bezanson, R., \& Franx, M. 2012, ApJ, 749, L10

Jog, C. J., \& Combes, F. 2009, Physics Reports, 471, 75

Johnston, E. J., Aragón-Salamanca, A., Merrifield, M. R., \& Bedregal, A. G. 2012, MNRAS, 422, 2590

Jungwiert, B., Combes, F., \& Palouš, J. 2001, A\&A, 376, 85

Katz, N., Weinberg, D. H., Hernquist, L., \& Miralda-Escude, J. 1996, ApJ, 457, L57

Kauffmann, G., White, S. D. M., \& Guiderdoni, B. 1993, MNRAS, 264, 201

Kauffmann, G., Colberg, J. M., Diaferio, A., \& White, S. D. M. 1999, MNRAS, 303, 188
Kaviraj, S., Schawinski, K., Devriendt, J. E. G., et al. 2007, ApJS, 173, 619

Keating, S.K., Abraham, R.G., Schiavon, R., et al. 2015, ApJ, 798,26

Kelvin, L. S., Driver, S. P., Robotham, A. S. G., et al. 2012, MNRAS, 421, 1007

Kereš, D., \& Hernquist, L. 2009, ApJ, 700, L1

Kereš, D., Katz, N., Weinberg, D. H., \& Davé, R. 2005, MNRAS, 363,2

Keselman, J. A., \& Nusser, A. 2012, MNRAS, 424, 1232

Khochfar, S., \& Burkert, A. 2006, A\&A, 445, 403

Khochfar, S., \& Silk, J. 2006, MNRAS, 370, 902

Kormendy, J., \& Kennicutt, R. C.. Jr. 2004, ARA\&A, 42, 603

Krajnović, D., Alatalo, K., Blitz, L., et al. 2013, MNRAS, 432, 1768

Kriek, M., van Dokkum, P. G., Franx, M., et al. 2006, ApJ, 649, L71

Kriek, M., van Dokkum, P. G., Franx, M., et al. 2008, ApJ, 677 , 219

Kroupa, P., Theis, C., \& Boily, C. M. 2005, A\&A, 431, 517

Kuntschner, H., \& Davies, R. L. 1998, MNRAS, 295, L29

Lange, R., Driver, S.P., Robotham, A.S.G., et al. 2015, MNRAS, 447, 2603

Laurikainen, E., Salo, H., Buta, R., Knapen, J. H., \& Comerón, S. 2010, MNRAS, 405, 1089

Lehner, N., Howk, J. C., Tripp, T. M., et al. 2013, ApJ, 770, 138

Li, C., Wang, E., Lin, L., et al. 2015, ApJ, in press (arXiv:1502.07040)

Licquia, T. C., \& Newman, J. A. 2014, ApJ, submitted (arXiv:1407.1078)

Liske, J., Lemon, D. J., Driver, S. P., Cross, N. J. G., \& Couch, W. J. 2003, MNRAS, 344, 307

López-Sanjuan, C., Le Fèvre, O., Ilbert, O., et al. 2012, A\&A, 548, A7

MacArthur L.A., González J.J., Courteau S. 2009, MNRAS, 395, 28

Maller, A. H., Katz, N., Kereš, D., Davé, R., \& Weinberg, D. H. 2006, ApJ, 647,763

Man, A.W.S., Zirm, A.W., Toft, S. 2015, ApJ, submitted (arXiv:1410.3479)

Mancini, C., Daddi, E., Renzini, A., et al. 2010, MNRAS, 401, 933

Mapelli, M., Rampazzo, R., Marino, A. 2015, A\&A, 575, A16

Marino, A., Bianchi, L., Rampazzo, R., et al. 2011, ApJ, 736, 154

Makarov, D., \& Karachentsev, I. 2011, MNRAS, 412, 2498

Makarov, D., Prugniel, P., Terekhova, N., Courtois, H., \& Vauglin, I. 2014, A\&A, 570, AA13

Martig, M., Bournaud, F., Teyssier, R., \& Dekel, A. 2009, ApJ, 707,250

McDermid, R.M. 2015, in Galaxy Masses as Constraints of Formation Models, IAU Symp. 311, M.Cappellari, S.Courteau (eds) (arXiv:1501.03927)

McLure, R. J., Pearce, H. J., Dunlop, J. S., et al. 2013, MNRAS, 428,1088

Merritt, D., Mikkola, S., \& Szell, A. 2007, ApJ, 671, 53

Metz, M., Kroupa, P., \& Jerjen, H. 2007,'MNRAS, 374, 1125

Möllenhoff, C., \& Heidt, J. 2001, A\&A, 368, 16

Moorthy, B.K., Holtzman, J.A. 2006, MNRAS, 371, 583

Muzzin, A., Labbé, I., Franx, M., et al. 2012, ApJ, 761, 142

Muzzin, A., Marchesini, D., Stefanon, M., et al. 2013, ApJ, 777, 18

Naab, T., \& Burkert, A. 2003, ApJ, 597, 893

Naab, T., Johansson, P. H., \& Ostriker, J. P. 2009, ApJ, 699, L178

Nair P., van den Bergh S., Abraham R.G. 2011, ApJ, 734, L31

Navarro J.F., Benz W. 1991, ApJ, 380, 320

Neichel, B., Hammer, F., Puech, M., et al. 2008, A\&A, 484, 159

Newman, A. B., Ellis, R. S., Bundy, K., \& Treu, T. 2012, ApJ, 746,162

Newman, A. B., Ellis, R. S., Treu, T., \& Bundy, K. 2010, ApJ, 717, L103

Nieto, J.-L., Capaccioli, M., \& Held, E. V. 1988, A\&A, 195, L1

Nipoti, C., Treu, T., Auger, M. W., \& Bolton, A. S. 2009, ApJ, $706, \mathrm{~L} 86$

Okuda, T., Kohno, K., Iguchi, S., \& Nakanishi, K. 2005, ApJ, 620,673

O'Sullivan, E., Combes, F., Hamer, S., et al. 2015, A\&A, 573, A111

Papovich, C., Dickinson, M., Giavalisco, M., Conselice, C. J., \& Ferguson, H. C. 2005, ApJ, 631, 101

Paudel, S., Lisker, T., Hansson, K. S. A., \& Huxor, A. P. 2014 MNRAS, 443, 446

Pawlowski, M. S., Famaey, B., Jerjen, H., et al. 2014, MNRAS, 442,2362

P érez, E., Cid Fernandes, R., Gonz alez Delgado, R. M., et al. 2013, ApJ, 764, L1

Pichon, C., Pogosyan, D., Kimm, T., et al. 2011, MNRAS, 418, 2493 
Pierini, D., Zibetti, S., Braglia, F. et al. 2008, A\&A, 483, 727 Planck Collaboration, Ade, P. A. R., Aghanim, N., et al. 2014, A\&A, 571, A16

Poggianti, B. M. 1997, A\&AS, 122, 399

oggianti, B. M., Calvi, R., Bindoni, D., et al. 2013a, ApJ, 762, 77

Poggianti, B. M., Moretti, A., Calvi, R., et al. 2013b, ApJ, 777, 125

Prescott, M.K.M., Martin, C.L., Dey, A. 2015, ApJ, 799, 62

Prieto, J., Jimenez, R., \& Haiman, Z. 2013, MNRAS, 436, 2301

Prochaska, J. X., Weiner, B., Chen, H.-W., Mulchaey, J., \& Cooksey, K. 2011, ApJ, 740, 91

Prochaska, J. X., Lau, M. W., \& Hennawi, J. F. 2014, ApJ, 796, 140

Querejeta, M., Eliche-Moral, M. C., Tapia, T., et al. 2015, A\&A, $573, \mathrm{~A} 78$

Ribaudo, J., Lehner, N., Howk, J. C., et al. 2011, ApJ, 743, 207

Riess, A. G., Macri, L., Casertano, S., et al. 2011, ApJ, 730, 119

Rigault, M., Aldering, G., Kowalski, M., et al. 2015, ApJ, in press (arXiv:1412.6501)

Robertson, B., Bullock, J. S., Cox, T. J., et al. 2006, ApJ, 645, 986

Rubin, V. C., Graham, J. A., \& Kenney, J. D. P. 1992, ApJ, 394, L9

Rubin, K. H. R., Prochaska, J. X., Koo, D. C., \& Phillips, A. C. 2012, ApJ, 747, L26

Ryan, R. E., Jr., McCarthy, P. J., Cohen, S. H., et al. 2012, ApJ, 749,53

Sage, L. J., Welch, G. A., \& Young, L. M. 2007, ApJ, 657, 232

Saha, K., Martinez-Valpuesta, I., \& Gerhard, O. 2012, MNRAS, 421, 333

Salim, S., Fang, J. J., Rich, R. M., Faber, S. M., \& Thilker, D. A. 2012, ApJ, 755, 105

Salomé, P., Combes, F., Edge, A. C., et al. 2006, A\&A, 454, 437

Sandage, A., \& Bedke, J. 1994, The Carnegie Atlas of Galaxies. Volumes I, II.,Carnegie Institution of Washington Publ., No. 638

Sandage, A., \& Tammann, G. A. 1981, Washington: Carnegie Institution, 1981

Santini, P., Maiolino, R., Magnelli, B., et al. 2014, A\&A, 562, A30

Saracco, P., Longhetti, M., \& Andreon, S. 2009, MNRAS, 392, 718

Scannapieco, C., White, S. D. M., Springel, V., \& Tissera, P. B. 2011, MNRAS, 417, 154

Schlafly, E. F., \& Finkbeiner, D. P. 2011, ApJ, 737, 103

Schombert, J., \& Smith, A. K. 2012, PASA, 29, 174

Schawinski, K., Thomas, D., Sarzi, M., et al. 2007, MNRAS, 382, 1415

Schawinski, K., Urry, C. M., Simmons, B. D., et al. 2014, MNRAS, 440,889

Scorza, C., \& van den Bosch, F. C. 1998, MNRAS, 300, 469

Scott, N., Davies, R. L., Houghton, R. C. W., et al. 2014, MNRAS, 441, 274

Scott, N., Graham, A. W., \& Schombert, J. 2013, ApJ, 768, 76

Seigar, M. S., Graham, A. W., \& Jerjen, H. 2007, MNRAS, 378, 1575

Seigar, M. S., \& James, P. A. 1998, MNRAS, 299, 672

Serra, P., Oosterloo, T., Morganti, R., et al. 2012, MNRAS, 422, 1835

Serra, P., Oser, L., Krajnović, D., et al. 2014, MNRAS, 444, 3388

Sérsic ,J.-L., 1968, Atlas de Galaxias Australes. Observatorio Astronomico Cordoba

Shen, S., Mo, H. J., White, S. D. M., et al. 2003, MNRAS, 343 , 978

Shih, H.-Y., \& Stockton, A. 2011, ApJ, 733, 45

Skrutskie, M. F., Cutri, R. M., Stiening, R., et al. 2006, AJ, 131, 1163

Springel, V., White, S. D. M., Jenkins, A., et al. 2005, Nature, 435,629

Steinmetz M., Navarro J.F. 2002, New Astronomy, 7, 155

Stewart, K. R., Brooks, A. M., Bullock, J. S., et al. 2013, ApJ, 769,74
Stocke, J. T., Keeney, B. A., Danforth, C. W., et al. 2013, ApJ, 763,148

Straatman, C. M. S., Labbé, I., Spitler, L. R., et al. 2014, ApJ, 783, L14

Szomoru, D., Franx, M., van Dokkum, P. G., et al. 2010, ApJ, $714, \mathrm{~L} 244$

Tacconi, L. J., Genzel, R., Smail, I., et al. 2008, ApJ, 680, 246

Taylor, E. N., Franx, M., Glazebrook, K., et al. 2010, ApJ, 720, 723

Taylor, E. N., Hopkins, A. M., Baldry, I. K., et al. 2011, MNRAS, 418, 1587

Temi, P., Brighenti, F., \& Mathews, W. G. 2007, ApJ, 660, 1215

Tempel, E., Guo, Q., Kipper, R., Libeskind, N.I. 2015, MNRAS, submitted (arXiv:1502.02046)

Thom, C., Werk, J. K., Tumlinson, J., et al. 2011, ApJ, 736, 1

Thom, C., Tumlinson, J., Werk, J. K., et al. 2012, ApJ, 758, L41

Thomas D., Davies R.L. 2006, MNRAS, 366, 510

Toft, S., van Dokkum, P., Franx, M., et al. 2007, ApJ, 671, 285

Tonry, J. L., Dressler, A., Blakeslee, J. P., et al. 2001, ApJ, 546, 681

Trujillo I. 2013, IAUS 295, 27

Trujillo, I., Cenarro, A. J., de Lorenzo-Cáceres, A., et al. 2009 , ApJ, 692, L118

Trujillo, I., Conselice, C. J., Bundy, K., et al. 2007, MNRAS, 382, 109

Trujillo I., Feulner, G., Goranova, Y., et al. 2006a, MNRAS, 373, L36

Trujillo, I., Förster Schreiber, N. M., Rudnick, G., et al. 2006b, ApJ, 650, 18

Trujillo, I., Rudnick, G., Rix, H.-W., et al. 2004, ApJ, 604, 521

De, T., Chattopadhyay, T., \& Chattopadhyay, A. K. 2014, PASA, 31, e047

Tully, R. B., Courtois, H. M., Dolphin, A. E., et al. 2013, AJ, 146, 86

Tumlinson, J., Thom, C., Werk, J. K., et al. 2013, ApJ, 777, 59

Valentinuzzi, T., Fritz, J., Poggianti, B. M., et al. 2010, ApJ, 712, 226

van den Bosch, R. C. E., Gebhardt, K., Gültekin, K., et al. 2012, Nature, 491,729

van der Wel, A., Rix, H.-W., Holden, B. P., Bell, E. F., \&

Robaina, A. R. 2009, ApJ, 706, L120

van der Wel A., Rix H.-W., Wuyts S., et al. 2011, ApJ, 730, 38

van der Wel, A., Franx, M., van Dokkum, P. G., et al. 2014, ApJ, 788,28

van Dokkum, P. G., Franx, M., Kriek, M., et al. 2008, ApJ, 677, L.

van Dokkum, P. G., Whitaker, K. E., Brammer, G., et al. 2010, ApJ, 709, 1018

van Dokkum, P. G., Leja, J., Nelson, E.J., et al. 2013, ApJ, 771, L35

van Dokkum, P. G., Bezanson, R., van der Wel, A., et al. 2014, ApJ, 791, 45

Wakker, B. P., \& Savage, B. D. 2009, ApJS, 182, 378

Wang, L., Zhu, W., Feng, L.-L., et al. 2014, MNRAS, 439, L85

Welker, C., Dubois, Y., Devriendt, J., et al. 2015, MNRAS, submitted (arXiv:1502.05053).

White, S. D. M., \& Frenk, C. S. 1991, ApJ, 379, 52

White, S. D. M., \& Rees, M. J. 1978, MNRAS, 183, 341

Wiklind, T., Combes, F., Henkel, C., \& Wyrowski, F. 1997, A\&A, 323,727

Yi, S. K., Yoon, S.-J., Kaviraj, S., et al. 2005, ApJ, 619, L111

York D.G., Adelman J., Anderson J.E., et al. 2000, AJ, 120, 1579

Young, L. M., Bureau, M., \& Cappellari, M. 2008, ApJ, 676, 317

Zhu, G., Ménard, B., Bizyaev, D., et al. 2014, MNRAS, 439, 3139

Zirm, A. W., van der Wel, A. Franx, M., et al. 2007, ApJ, 656, 66

Zoccali, M., Lecureur, A., Barbuy, B., et al. 2006, A\&A, 457, L1

Zolotov, A., Dekel, A., Mandelker, N., et al. 2014, MNRAS, submitted (arXiv:1412.4783) 